\title{
Variational MultiScale based dissipation models for the estimation of Atmospheric Seeing
}

\author{
Joan Baiges ${ }^{* 1,2}$ and Ramon Codina ${ }^{2,1}$ \\ ${ }^{1}$ Centre Internacional de Mètodes Numèrics a l'Enginyeria (CIMNE), \\ Edifici C1, Campus Nord UPC C/ Gran Capità S/N 08034 Barcelona, Spain \\ ${ }^{2}$ Universitat Politècnica de Catalunya, Jordi Girona 1-3, Edifici C1, 08034 Barcelona, Spain \\ *jbaiges@cimne.upc.edu, Telf: +0034934016206
}

September 9, 2014

\begin{abstract}
In this work we present a numerical model for the estimation of atmospheric seeing in observation sites. The particularity of the method is that it is based on a Variational MultiScale turbulence model, its main feature being that the numerical mechanisms which are used to deal with stability issues (convection and the inf-sup condition for incompressible flows) do also take care of the modeling of turbulence. Based on this turbulence model, we develop the expressions for the viscous and thermal dissipations, $\epsilon_{\text {num }}$ and $\chi_{\text {num }}$, which are later used for evaluating the constant of structure of the refraction index $C_{n}^{2}$ following the classical model developed by Tatarski. Numerical examples show the behavior of the proposed numerical scheme when applied to turbulent flow practical cases, which include a convective boundary layer, the flow inside a transfer optics room, and a telescope enclosure.
\end{abstract}

\section{Contents}

1 Introduction $\quad 2$

2 Incompressible Navier-Stokes equations $\quad 4$

2.1 Problem statement . . . . . . . . . . . . . . . . . . . 4

2.2 Finite element approximation . . . . . . . . . . . . . . . 4

2.3 Turbulent viscous dissipation for the OSS Navier-Stokes equations . . . . . . 7

2.4 Discretization in time . . . . . . . . . . . . . . . . . 9

2.5 Fractional step splitting . . . . . . . . . . . . . . . . . . 9

3 Heat transfer equation $\quad 10$

3.1 Problem statement . . . . . . . . . . . . . . . . . . . . 10

3.2 Finite element approximation . . . . . . . . . . . . . . . . . 11

3.3 Turbulent thermal dissipation for the OSS heat transfer equation . . . . . . . . 11

3.4 Discretization in time . . . . . . . . . . . . . . . . 12

4 Atmospheric seeing parameters $\quad 12$ 
5 Numerical examples $\quad 14$

5.1 Convective boundary layer . . . . . . . . . . . . . . . . . . . 14

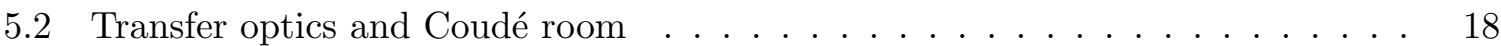

5.3 Flow around a telescope enclosure $\ldots \ldots \ldots \ldots \ldots$

6 Conclusions 23

\section{Introduction}

In the observation of the universe by using ground based telescope facilities, the effect of atmospheric turbulence in the quality of the obtained data is of great importance. Especially when the diameter of the telescope is large, the optical distortion due to turbulent effects can become significant enough to prevent the observation device to reach its maximum theoretical resolution. This is the reason why extensive efforts have been done in several directions:

- The development of adaptive optics mechanisms which allow to correct the phase distortion effects. This is achieved by dividing the main mirror of the telescope into smaller mirrors and allowing these sub-mirrors to move independently, which allows to obtain a corrected image. This approach has been successfully applied to the new generation telescopes, but the number of mirrors the main mirror needs to be composed of and the speed at which these mirrors need to change their configuration directly depend on the atmospheric turbulence intensity.

- The selection of observation sites which provide optimal atmospheric conditions. Very specific locations around the world have turned out to be optimal observation sites. Amongst them, the Canary Islands in Spain, Hawaii in the USA or the Atacama desert in Chile concentrate some of the largest telescopes in the world thanks to their pristine seeing conditions.

- The design of telescope facilities which minimize the amount of turbulence of the aerodynamic flow around them. Minimizing the small eddy oscillations in the air flow around the telescope mirrors results in less optical distortion, which means that the corrections to be done by the adaptive mechanism are lesser, which in turn results in better acquired images. This is especially true in the case of solar telescopes, where the large temperature gradients at the ground surface due to radiation effects have a critical impact in the condition of collected data.

It is clear that being able to evaluate the suitability of an observation site or a telescope facility design prior to the telescope construction is key for getting the best possible performance out of the telescope. Also, it is convenient to be able to quantify the characteristics of the adaptive optics mechanism to be installed in the telescope beforehand. Due to this, several efforts have been devoted recently to the quantification of atmospheric seeing parameters through numerical simulation.

One of the first numerical models for the quantification of the temperature structure function was presented in [20]. In [29, 30] an atmospheric Meso-Nh numerical model was used to compute atmospheric seeing in an observation site in Cerro Paranal, Chile. Soon after, the same methodology was applied to the simulation of another observation site at the Roque de Los Muchachos, Canary Islands, Spain [31]. In [7, 8, 6] a Large Eddy Simulation (LES) model run on a weather forecast simulation software was used to characterize near-surface optical turbulence under different climatic conditions in observation sites. A seeing model which makes 
use of the turbulent kinetic energy provided by a planetary boundary layer simulation software was used to compute seeing in Mauna Kea, Hawaii in [10, 9]. In [22] a weather forecasting model was used together with the statistical parametrization of the refraction index structure constant $C_{n}^{2}$ presented in [35] in order to forecast the seeing conditions in the Roque de Los Muchachos, Canary Islands, Spain. A numerical weather prediction tool was used in [1] to compute various turbulence parameters which allow to characterise $C_{n}^{2}$ in the islands of Maui and the Big Island, Hawaii, and a similar methodology was presented in [21].

All of these methodologies have in common the use of numerical weather prediction tools for the simulation of seeing conditions, where the expression for $C_{n}^{2}$ is derived from different mesoscale turbulence models. In [13] we presented a strategy for the computation of atmospheric seeing parameters, which, to our knowledge, is the first computational method capable of simulating seeing conditions at a local level; that is, by using a finite element computational fluid dynamics simulation with a resolution ranging from decimeters to few meters instead of using mesoscale simulation tools with a coarse resolution. The proposed model for the computation of the refraction index structure function $C_{n}^{2}$ is based on a Large Eddy Simulation of the incompressible flow and temperature fields, and has been successfully applied to the design phase of the Advance Technology Solar Telescope (ATST) [28] and the European Solar Telescope $(\mathrm{EST})[3,5]$.

In this work we present a model for the numerical simulation of the $C_{n}^{2}$ function which is computed from a variational multiscale (VMS) [26] based turbulence model. For finite element analysis, the basic idea of VMS is to split the unknowns into their finite-element part and a subgrid scale component, the subscale. The approximation adopted for the subscale defines the numerical model. The interesting feature about VMS is that it is capable of providing, at the same time, a numerical stabilization mechanism for the studied equations (in this case, the incompressible Navier-Stokes equations) and a turbulence model which takes into account the under-resolved scales (those which cannot be captured by the finite element mesh). This has been studied in several works $[27,18,14,15,23]$ with successful results. The advantage of using this kind of approach is that there is no interference between the numerical stabilization and the turbulence models because both issues are taken care of by the numerical subgrid scales.

The method used in the present work is the Orthogonal Subgrid Scale VMS method (OSS). Its particularity is that it models the numerical subscales in a rich manner: the subscales are considered to be transient in time, non-linear, and orthogonal to the finite element space. A theoretical analysis of the orthogonal-subscales VMS turbulence model is presented in [24], and an extensive campaign of numerical experiments is presented in [19]. The conclusions of these experiments are that VMS turbulence models can provide an accurate representation of turbulent phenomena at a competitive computational cost, with the particular feature that the turbulence model arises from numerical reasoning only. In this work we use the kinetic energy and thermal turbulent dissipations which arise from the OSS turbulence model in order to quantify the refraction index structure function.

The paper is organized as follows. The finite element approximation of the incompressible Navier-Stokes equations is presented in Section 2, where the variational multiscale method and the kinetic energy turbulent dissipation are also presented for this model problem. In Section 3, the finite element approximation of the heat transfer equation is presented, together with the OSS formulation and the thermal energy turbulent dissipation expressions for the temperature problem. In Section 4 the numerical model for the computation of seeing quantities arising from the OSS dissipations is presented. This allows us to compute the refraction index structure function as well as optical magnitudes of interest such as the Fried parameter and the Greenwood frequency. Numerical examples illustrate the performance of the proposed numerical 
method in Section 5. Finally, some conclusions close the paper in Section 6 .

\section{Incompressible Navier-Stokes equations}

\subsection{Problem statement}

In this section we summarize the Orthogonal Subgrid-Scale (OSS) approach applied to the incompressible Navier-Stokes equations described in [11]. Let us consider the transient incompressible Navier-Stokes equations, which consist of finding $\boldsymbol{u}: \Omega \times(0, T) \longrightarrow \mathbb{R}^{3}$ and $p: \Omega \times(0, T) \longrightarrow \mathbb{R}$ such that:

$$
\begin{aligned}
& \partial_{t} \boldsymbol{u}-2 \nu \nabla \cdot \nabla^{s} \boldsymbol{u}+\boldsymbol{u} \cdot \nabla \boldsymbol{u}+\nabla p=\boldsymbol{f} \quad \text { in } \Omega, \\
& \nabla \cdot \boldsymbol{u}=0 \quad \text { in } \Omega \text {, } \\
& \boldsymbol{u}=\overline{\boldsymbol{u}} \text { on } \Gamma_{D}, \\
& -p \boldsymbol{n}+\nu \boldsymbol{n} \cdot \nabla \boldsymbol{u}=\mathbf{0} \quad \text { on } \Gamma_{N} .
\end{aligned}
$$

for $t>0$, where $\partial_{t} \boldsymbol{u}$ is the local time derivative of the velocity field and $\nabla^{s} \boldsymbol{u}=\frac{1}{2}\left((\nabla \boldsymbol{u})^{T}+\nabla \boldsymbol{u}\right)$. $\Omega \subset \mathbb{R}^{3}$ is a bounded domain, $\nu$ is the viscosity, and $f$ the given source term. $\Gamma_{D}$ is the Dirichlet boundary, where velocity boundary conditions are applied. In the case of the numerical simulation of aerodynamics of telescopes, it corresponds to the inflow boundary and the ground and telescope surfaces. $\Gamma_{N}$ is the Neumann boundary, where conditions on the value of the tractions are applied. In aerodynamics of telescopes simulations, it corresponds to the outflow boundary. Appropriate initial conditions have to be appended to this problem. In the numerical examples section, the source term $f$ is due to the Boussinesq buoyancy forces, which appear due to temperature gradients and are of the form:

$$
\boldsymbol{f}=\alpha \boldsymbol{g}\left(\theta-\theta_{0}\right),
$$

where $\alpha$ is the thermal expansion coefficient, $\boldsymbol{g}$ is the gravity acceleration vector, $\theta_{0}$ is a reference temperature and $\theta$ the temperature of the fluid.

\section{$2.2 \quad$ Finite element approximation}

Let us now consider the finite element approximation of equations (1)-(4). We define $V=$ $H^{1}(\Omega)^{d}$, and $V_{0}=\left\{\boldsymbol{v} \in V \mid \boldsymbol{v}=\mathbf{0}\right.$ on $\left.\Gamma_{D}\right\}$. Let also $Q=L^{2}(\Omega)$ and $\mathcal{D}^{\prime}(0, T ; Q)$ be the distributions in time with values in $Q$. The variational problem consists of finding $[\boldsymbol{u}, p] \in$ $L^{2}(0, T ; V) \times \mathcal{D}^{\prime}(0, T ; Q)$ such that:

$$
\begin{array}{rr}
\left(\boldsymbol{v}, \partial_{t} \boldsymbol{u}\right)+\langle\boldsymbol{v}, \boldsymbol{u} \cdot \nabla \boldsymbol{u}\rangle+2 \nu\left(\nabla^{s} \boldsymbol{v}, \nabla^{s} \boldsymbol{u}\right)-(p, \nabla \cdot \boldsymbol{v})=\langle\boldsymbol{v}, \boldsymbol{f}\rangle & \forall \boldsymbol{v} \in V_{0} \\
(q, \nabla \cdot \boldsymbol{u})=0 & \forall q \in Q
\end{array}
$$

with

$$
\boldsymbol{u}=\overline{\boldsymbol{u}} \quad \text { on } \Gamma_{D}
$$

Here, $(\cdot, \cdot)$ stands for the $L^{2}(\Omega)$ inner product and $\langle\cdot, \cdot\rangle$ for the integral of the product of two functions, not necessarily in $L^{2}(\Omega)$. Let us also define

$$
B([\boldsymbol{v}, q],[\boldsymbol{a} ; \boldsymbol{u}, p]):=\langle\boldsymbol{v}, \boldsymbol{a} \cdot \nabla \boldsymbol{u}\rangle+2 \nu\left(\nabla^{s} \boldsymbol{v}, \nabla^{s} \boldsymbol{u}\right)-(p, \nabla \cdot \boldsymbol{v})+(q, \nabla \cdot \boldsymbol{u}),
$$


where $\boldsymbol{a}$ represents the convective velocity. When the Galerkin finite element approximation is used, it is well known that the semilinear form $B$ suffers from stability issues due to the convective nature of the flow, but also requires a compatibility between the velocity and pressure approximation spaces due to the classical LBB inf-sup condition.

Many stabilization methods have been developed to deal with these issues in the past decades. Amongst them, one of the stabilization approaches which has received more interest in recent years is the variational multiscale (VMS) method. The most interesting feature of VMS is that the stabilization terms are not derived from purely numerical reasons, but are motivated from a physical point of view. This physical approach comes from the introduction of a decomposition of a general solution of the problem of interest into its finite element part and the part of the solution which cannot be captured by the finite element mesh. In order to introduce the subscale concept in the weak form (5), let us consider the finite element partition $\mathcal{T}_{h}:=\{K\}$ defined over domain $\Omega, h$ denoting the element size. To simplify the exposition, we consider $\mathcal{T}_{h}$ quasi-uniform. From this partition we construct the finite element spaces $V_{h} \subset V$, $Q_{h} \subset Q$. We will seek for approximate solutions $\boldsymbol{u}_{h} \in C^{1}\left(0, T ; V_{h}\right)$ and $p_{h} \in C^{0}\left(0, T ; Q_{h}\right)$.

The variational multiscale method is derived by introducing the subscales space for the velocity field $\tilde{V}$ such that:

$$
V=V_{h} \oplus \tilde{V}
$$

This implies that the velocity solution field and the velocity test functions can also be decomposed into the finite element part and the fine scale part:

$$
\begin{aligned}
& \boldsymbol{u}=\boldsymbol{u}_{h}+\tilde{\boldsymbol{u}} \\
& \boldsymbol{v}=\boldsymbol{v}_{h}+\tilde{\boldsymbol{v}} .
\end{aligned}
$$

The same decomposition can be applied to the pressure field by introducing the pressure subscales space $\tilde{Q}$, as described in [12]:

$$
\begin{aligned}
Q & =Q_{h} \oplus \tilde{Q} \\
p & =p_{h}+\tilde{p} \\
q & =q_{h}+\tilde{q} .
\end{aligned}
$$

Also, we consider the subscales to vanish on the element boundaries, although their contribution in the element faces can also be taken into account, as described in [17]. Introducing this splitting in equation (5), and after integrating by parts some of terms, the discrete variational problem we obtain is to find $\left[\boldsymbol{u}_{h}, p_{h}\right] \in V_{h} \times Q_{h}$, for each $t \in[0, T]$ and $\tilde{\boldsymbol{u}} \in \tilde{V}, \tilde{p} \in \tilde{Q}$ for each $t \in[0, T]$, such that:

$$
\begin{aligned}
\left(\boldsymbol{v}_{h}, \partial_{t} \boldsymbol{u}_{h}\right)+B\left(\left[\boldsymbol{v}_{h}, q_{h}\right],\left[\boldsymbol{u} ; \boldsymbol{u}_{h}, p_{h}\right]\right) & \\
+\sum_{K}\left\langle\boldsymbol{v}_{h}, \partial_{t} \tilde{\boldsymbol{u}}\right\rangle_{K}+\sum_{K}\left\langle-\nu \Delta \boldsymbol{v}_{h}-\boldsymbol{u} \cdot \nabla \boldsymbol{v}_{h}-\nabla q_{h}, \tilde{\boldsymbol{u}}\right\rangle_{K}-\sum_{K}\left\langle\nabla \cdot \boldsymbol{v}_{h}, \tilde{p}\right\rangle_{K} & =\left\langle\boldsymbol{v}_{h}, \boldsymbol{f}\right\rangle, \\
\sum_{K}\left\langle\tilde{\boldsymbol{v}}, \partial_{t} \boldsymbol{u}_{h}-\nu \Delta \boldsymbol{u}_{h}+\boldsymbol{u} \cdot \nabla \boldsymbol{u}_{h}+\nabla p_{h}\right\rangle_{K} & \\
+\sum_{K}\left\langle\tilde{\boldsymbol{v}}, \partial_{t} \tilde{\boldsymbol{u}}-\nu \Delta \tilde{\boldsymbol{u}}+\boldsymbol{u} \cdot \nabla \tilde{\boldsymbol{u}}+\nabla \tilde{p}\right\rangle_{K} & =\langle\tilde{\boldsymbol{v}}, \boldsymbol{f}\rangle, \\
\sum_{K}\left\langle\tilde{q}, \nabla \cdot \boldsymbol{u}_{h}\right\rangle_{K}+\sum_{K}\langle\tilde{q}, \nabla \cdot \tilde{\boldsymbol{u}}\rangle_{K} & =0,
\end{aligned}
$$


for all test functions $\boldsymbol{v}_{h}, q_{h}, \tilde{\boldsymbol{v}}, \tilde{q}$, and where $\langle\cdot, \cdot\rangle_{K}$ stands for the integral of the product of two functions in $K$. Note that, for incompressible flows, $2 \nabla \cdot \nabla^{s} \boldsymbol{u}=\Delta \boldsymbol{u}$. Equation (8) is the subscales equation, which will be used to provide a closure for the expressions of the velocity and pressure subscales, $\tilde{\boldsymbol{u}}$ and $\tilde{p}$. Following the steps in [18] we first consider the equation for the velocity subscales, omitting the contribution of the pressure subscales. This allows us to formulate a simpler method, and it implicitly assumes that the velocity subscales are due to the residual of the momentum equations, instead of being driven by the incompressibility constraint. The second term in equation (8) can be modeled as follows:

$$
\sum_{K}\langle\tilde{\boldsymbol{v}},-\nu \Delta \tilde{\boldsymbol{u}}+\boldsymbol{u} \cdot \nabla \tilde{\boldsymbol{u}}\rangle_{K} \simeq \tau_{K}^{-1}\langle\tilde{\boldsymbol{v}}, \tilde{\boldsymbol{u}}\rangle_{K}, \quad \tau_{K}^{-1}=\left(c_{1} \frac{\nu}{h^{2}}+c_{2} \frac{|\boldsymbol{u}|}{h}\right),
$$

where $c_{1}$ and $c_{2}$ are algorithmic constants. The approximation of the subscales operator as a scalar term times the product $\langle\tilde{\boldsymbol{v}}, \tilde{\boldsymbol{u}}\rangle$ can be justified by means of a Fourier analysis of the problem for the subscales [12]. Replacing this expression in the equation for the subscales we obtain:

$$
\sum_{K}\left\langle\tilde{\boldsymbol{v}}, \partial_{t} \boldsymbol{u}_{h}-\nu \Delta \boldsymbol{u}_{h}+\boldsymbol{u} \cdot \nabla \boldsymbol{u}_{h}+\nabla p_{h}\right\rangle_{K}+\sum_{K}\left\langle\tilde{\boldsymbol{v}}, \partial_{t} \tilde{\boldsymbol{u}}\right\rangle_{K}+\sum_{K} \tau_{K}^{-1}\langle\tilde{\boldsymbol{v}}, \tilde{\boldsymbol{u}}\rangle_{K}=\langle\tilde{\boldsymbol{v}}, \boldsymbol{f}\rangle .
$$

Equation (11) yields an expression for the subscales in the element interiors in terms of the finite element component, as long as $\tilde{V}$ is approximated by a space of discontinuous functions:

$$
\partial_{t} \tilde{\boldsymbol{u}}+\tau_{K}^{-1} \tilde{\boldsymbol{u}}=P_{\tilde{V}}\left(\boldsymbol{f}-\left(\partial_{t} \boldsymbol{u}_{h}-\nu \Delta \boldsymbol{u}_{h}+\boldsymbol{u} \cdot \nabla \boldsymbol{u}_{h}+\nabla p_{h}\right)\right),
$$

where $P_{\tilde{V}}$ denotes the projection onto the subscales space. Equation (12) implicitly defines a $\tilde{\boldsymbol{u}}$ as a function of $\boldsymbol{u}_{h}, p_{h}$ and $t$ :

$$
\tilde{\boldsymbol{u}}=\phi_{\tilde{\boldsymbol{u}}}\left(\boldsymbol{u}_{h}, p_{h}, t\right) .
$$

There are several possible choices for the space of subscales, which yield different projection operators $P_{\tilde{V}}$. A typical choice is $P_{\tilde{V}}=I$, which results in the Algebraic Subgrid-Scale formulation (ASGS). The formulation we favor in our VMS model is to take the subscale space to be orthogonal to the finite element space:

$$
\tilde{V}=V_{h}^{\perp} \cap V,
$$

and as a consequence:

$$
P_{\tilde{V}}=I-P_{h},
$$

where $P_{h}$ is the $L^{2}(\Omega)$ projection onto the finite element space. This formulation is called the Orthogonal Subgrid Scale (OSS) formulation. It usually results in sharper, less diffusive solutions than the ASGS method.

This provides the required expression for the subscales in terms of the finite element unknowns. Note that equation (12) involves the time derivative of the velocity subscales. As a consequence, the subscales will need to be tracked in time and a time discretization scheme for them will be required. Note also that the velocity in all the convective terms is $\boldsymbol{u}=\boldsymbol{u}_{h}+\tilde{\boldsymbol{u}}$, leading to a non-linear expression for the velocity subscales.

The pressure subscales can be modeled as:

$$
\tilde{p}=\phi_{\tilde{p}}\left(\boldsymbol{u}_{h}\right):=\tau_{p} \tau_{K}^{-1} P_{\tilde{Q}}\left(\nabla \cdot \boldsymbol{u}_{h}\right), \quad \tau_{p}=c_{3} h^{2},
$$


following a procedure similar to the one described for the velocity subscales in equation (10). Again, $\tilde{Q}$ is taken to be orthogonal to $Q$ :

$$
\tilde{Q}=Q_{h}^{\perp} \cap Q .
$$

Note that both equation (12) and (14) need to be solved at the numerical integration points, since the subscales are needed there to compute (numerically) the integrals appearing in (7).

This final formulation of the incompressible Navier-Stokes equations can be proven to be stable for a proper choice of the stabilization constants $c_{1}$ and $c_{2}$. Equally important, the motivation for the stabilizing terms has arised from taking into account the contribution of the part of the solution which cannot be captured by the finite element mesh. This is the reason why many authors $[27,18,14,15,23]$ have decided to use these numerical subscales not only as a stabilizing mechanism, but also as a model for the physics undergoing below the spatial and temporal resolution of the finite element mesh. In the case of the Navier-Stokes equations this corresponds to the modeling of turbulence.

\subsection{Turbulent viscous dissipation for the OSS Navier-Stokes equations}

Let us now focus on the numerical dissipation (and also turbulent, if we attend to the physical meaning of the subscales) of the scheme. We define the kinetic energy per unit volume inside the computational domain as:

$$
W:=\frac{1}{2} \rho|\boldsymbol{u}|^{2} .
$$

The balance of kinetic energy for the continuous problem can be obtained by taking the test function $\boldsymbol{v}$ equal to the velocity $\boldsymbol{u}$, the test function $q$ equal to the pressure $p$, and multiplying (5) and (6) by the density $\rho$. After some manipulations we arrive to:

$$
\frac{\partial}{\partial t} \int_{\Omega} W+\int_{\partial \Omega} \boldsymbol{n} \cdot \boldsymbol{u} W=\langle\boldsymbol{u}, \rho \boldsymbol{f}\rangle-2 \mu\left(\nabla^{s} \boldsymbol{u}, \nabla^{s} \boldsymbol{u}\right),
$$

where $\mu$ is the dynamic viscosity. Equation (15) is saying that the variation of kinetic energy in the computational volume $\frac{\partial}{\partial t} \int_{\Omega} W$ is due to the flux of kinetic energy through the domain boundaries $\int_{\partial \Omega} \boldsymbol{n} \cdot \boldsymbol{u} W$, the increment of kinetic energy due to body forces $\langle\boldsymbol{u}, \rho \boldsymbol{f}\rangle$ (external power) and the decrement of kinetic energy due to the viscous dissipation $\epsilon=\mu(\nabla \boldsymbol{u}, \nabla \boldsymbol{u})$. The discrete counterpart of (15) is obtained by taking $\boldsymbol{v}_{h}=\boldsymbol{u}_{h}, q_{h}=p_{h}$ in (7):

$$
\begin{array}{r}
\frac{\partial}{\partial t} \int_{\Omega} W_{h}+\int_{\partial \Omega} \boldsymbol{n} \cdot\left(\boldsymbol{u}_{h}+\tilde{\boldsymbol{u}}\right) W_{h}=\left\langle\boldsymbol{u}_{h}, \rho \boldsymbol{f}\right\rangle-2 \mu\left(\nabla^{s} \boldsymbol{u}_{h}, \nabla^{s} \boldsymbol{u}_{h}\right)-\sum_{K}\left\langle\rho \boldsymbol{u}_{h}, \partial_{t} \tilde{\boldsymbol{u}}\right\rangle_{K} \\
+\sum_{K}\left\langle\mu \Delta \boldsymbol{u}_{h}+\rho\left(\boldsymbol{u}_{h}+\tilde{\boldsymbol{u}}\right) \cdot \nabla \boldsymbol{u}_{h}+\rho \nabla p_{h}, \tilde{\boldsymbol{u}}\right\rangle_{K}+\sum_{K}\left\langle\rho \nabla \cdot \boldsymbol{u}_{h}, \tilde{p}\right\rangle_{K},
\end{array}
$$

with:

$$
W_{h}:=\frac{1}{2} \rho\left|\boldsymbol{u}_{h}\right|^{2} .
$$

The terms involving the subscales in the right-hand side of equation (16) constitute the viscous numerical dissipation:

$$
D_{\text {num }}:=\sum_{K} \int_{K} \epsilon_{\text {num }}
$$




$$
\epsilon_{\mathrm{num}}:=\rho \boldsymbol{u}_{h} \cdot \partial_{t} \tilde{\boldsymbol{u}}-\left(\mu \Delta \boldsymbol{u}_{h}+\rho\left(\boldsymbol{u}_{h}+\tilde{\boldsymbol{u}}\right) \cdot \nabla \boldsymbol{u}_{h}+\rho \nabla p_{h}\right) \cdot \tilde{\boldsymbol{u}}-\rho \nabla \cdot \boldsymbol{u}_{h} \tilde{p} .
$$

On the other hand, we have the dissipation due to the finite element part of the solution:

$$
\begin{aligned}
D_{h} & =\int_{\Omega} \epsilon_{h}, \\
\epsilon_{h}: & =2 \mu\left|\nabla^{s} \boldsymbol{u}\right|^{2} .
\end{aligned}
$$

$\epsilon_{\text {num }}$ appears due to the contribution of the stabilization terms and, at the same time, it is taking into account the dissipation due to the subscales. In order to show this, let us study a simplified problem where we neglect the contribution of the pressure subscales $\tilde{p}$. Let us also neglect the contribution of $\mu \Delta \boldsymbol{u}_{h}$ (which is reasonable if highly turbulent flows are considered, or exact if a linear interpolation space is chosen for $\boldsymbol{u}_{h}$ ). Furthermore, we take into account that, if $\tilde{V} \subset V_{h}^{\perp} \cap V$, then:

$$
\sum_{K}\left\langle\rho \boldsymbol{u}_{h}, \partial_{t} \tilde{\boldsymbol{u}}\right\rangle_{K}=0
$$

Let us also define the kinetic energy of $\tilde{\boldsymbol{u}}$ :

$$
\tilde{W}:=\frac{1}{2} \rho|\tilde{\boldsymbol{u}}|^{2}
$$

the dissipation of $\tilde{\boldsymbol{u}}$ :

$$
\tilde{D}=\sum_{K} \int_{K} \tilde{\epsilon}, \quad \tilde{\epsilon}=\tau_{K}^{-1}|\tilde{\boldsymbol{u}}|^{2},
$$

and the external power on $\boldsymbol{u}_{h}$ and $\tilde{\boldsymbol{u}}$ :

$$
\mathcal{P}_{h}:=\left\langle\boldsymbol{u}_{h}, \rho \boldsymbol{f}\right\rangle, \quad \tilde{\mathcal{P}}:=\langle\tilde{\boldsymbol{u}}, \rho \boldsymbol{f}\rangle .
$$

Let us multiply (8) by $\rho$ and take $\tilde{\boldsymbol{v}}=\tilde{\boldsymbol{u}}$. In this case from (8) and (16) we have:

$$
\begin{aligned}
\frac{\partial}{\partial t} \int_{\Omega} W_{h}+\int_{\partial \Omega} \boldsymbol{n} \cdot \boldsymbol{u} W_{h}+D_{h}+D_{\text {num }} & =\mathcal{P}_{h} \\
\frac{\partial}{\partial t} \int_{\Omega} \tilde{W}+\tilde{D}-D_{\text {num }} & =\tilde{\mathcal{P}}
\end{aligned}
$$

It can be seen from equations (18)-(19) that $\epsilon_{\text {num }}$ is in charge for the transfer of energy from the large (finite element) scales to the subgrid scales. Due to this, $\epsilon_{\text {num }}$ can be understood as the dissipation caused by the turbulent effects of the flow (see [19, 24]).

Another interesting observation can be done if we plug the equation for the subscales (12) into the expression for $\epsilon_{\text {num }}$ (17) (again taking into account the orthogonality of the subscales and neglecting $\tilde{p}$ and $\left.\mu \Delta \boldsymbol{u}_{h}\right)$ :

$$
\begin{aligned}
D_{\text {num }}= & \rho \sum_{K} \tau_{K}\left\langle P_{\tilde{V}}\left(\boldsymbol{u} \cdot \nabla \boldsymbol{u}_{h}+\nabla p_{h}\right), P_{\tilde{V}}\left(\boldsymbol{u} \cdot \nabla \boldsymbol{u}_{h}+\nabla p_{h}\right)\right\rangle_{K} \\
& +\rho \sum_{K} \tau_{K}\left\langle P_{\tilde{V}}\left(\boldsymbol{u} \cdot \nabla \boldsymbol{u}_{h}+\nabla p_{h}\right), \partial_{t} \tilde{\boldsymbol{u}}\right\rangle_{K} .
\end{aligned}
$$

The first term in the right-hand side of (20) is always positive. The second term only appears if dynamic subgrid scales are considered. It is shown in [16] that it can be locally (in space and time) negative, although the average in time of $\epsilon_{\text {num }}$ is proved to be positive. Locally 
negative values of $\epsilon_{\text {num }}$ represent a transfer of energy from the small scales to the large scales, a phenomenon known as backscatter, which is observed in physical turbulent flows. After the decomposition of the unknowns into the contributions of the finite element scales and the subgrid scales, taking into account the temporal derivatives of the subscales allows the OSS method to model backscatter. Let us stress that this backscatter model arises from the numerical decomposition of the unknown and not from a physical modeling of the phenomena. See the numerical examples section where dissipation values and their average are shown for a convective boundary layer case. Another numerical example can be found in [16].

Finally, the total pointwise dissipation which accounts for the numerical and large scale dissipation:

$$
\epsilon=\epsilon_{h}+\epsilon_{\text {num }}
$$

is going to be used for the computation of quality of seeing estimators.

\subsection{Discretization in time}

For the discretization in time, we rely on a finite difference time discretization. We consider a uniform partition of $[0, T]$ of size $\delta t$. We denote a time dependent function $f$ approximated at $t^{n}=n \delta t$ as $f^{n}$ and $\delta_{t} f^{n}$ the approximation to $\partial_{t} f$ at $t^{n}$. Introducing this notation, the time discrete problem is: find $\left[\boldsymbol{u}_{h}, p_{h}\right] \in V_{h} \times Q_{h}$, for each $t \in[0, T]$ and $\tilde{\boldsymbol{u}} \in \tilde{V}, \tilde{p} \in \tilde{Q}$ for each $t \in[0, T]$, such that:

$$
\begin{aligned}
& \left(\boldsymbol{v}_{h}, \delta_{t} \boldsymbol{u}_{h}^{n+1}\right)+B\left(\left[\boldsymbol{v}_{h}, q_{h}\right],\left[\boldsymbol{u}^{n+1} ; \boldsymbol{u}_{h}^{n+1}, p_{h}^{n+1}\right]\right)+\sum_{K}\left\langle\boldsymbol{v}_{h}, \delta_{t} \tilde{\boldsymbol{u}}^{n+1}\right\rangle_{K} \\
& +\sum_{K}\left\langle-\nu \Delta \boldsymbol{v}_{h}-\boldsymbol{u}^{n+1} \cdot \nabla \boldsymbol{v}_{h}-\nabla q_{h}, \tilde{\boldsymbol{u}}^{n+1}\right\rangle_{K}-\sum_{K}\left\langle\nabla \cdot \boldsymbol{v}_{h}, \tilde{p}^{n+1}\right\rangle_{K}=\left\langle\boldsymbol{v}_{h}, \boldsymbol{f}^{n+1}\right\rangle .
\end{aligned}
$$

In order to approximate $\delta_{t} \boldsymbol{u}_{h}^{n+1}$, a second order backward differences scheme is used:

$$
\delta_{t} \boldsymbol{u}_{h}^{n+1}=\frac{\frac{3}{2} \boldsymbol{u}_{h}^{n+1}-2 \boldsymbol{u}_{h}^{n}+\frac{1}{2} \boldsymbol{u}_{h}^{n-1}}{\delta t} .
$$

On the other hand, a first order backward Euler scheme is used to integrate the subscales in time:

$$
\delta_{t} \tilde{\boldsymbol{u}}^{n+1}=\frac{\tilde{\boldsymbol{u}}^{n+1}-\tilde{\boldsymbol{u}}^{n}}{\delta t}
$$

This is sufficient for the approximation of the subscales, since:

$$
\tilde{\boldsymbol{u}}=\mathcal{O}\left(\tau_{K}\left(\boldsymbol{f}-\left(\partial_{t} \boldsymbol{u}_{h}-\nu \Delta \boldsymbol{u}_{h}+\boldsymbol{u} \cdot \nabla \boldsymbol{u}_{h}+\nabla p_{h}\right)\right)\right.
$$

and $\tau_{K}=\mathcal{O}(\delta t)$.

\subsection{Fractional step splitting}

In order to minimize the computational time inverted in solving (22), we rely on a fractional step splitting technique. This decomposes the solution of the monolithic system into the solution of several simpler problems: a convection-diffusion equation for the velocity unknown, a pressurepoisson equation and a projection step. We start by noting that, for the choice $\tilde{V}=V_{h}^{\perp} \cap V$ :

$$
\sum_{K}\left\langle\boldsymbol{v}_{h}, \delta_{t} \tilde{\boldsymbol{u}}^{n+1}\right\rangle_{K}=0
$$


We introduce an intermediate velocity $\hat{\boldsymbol{u}}_{h}$, for which we consider:

$$
\begin{aligned}
\hat{\boldsymbol{u}}_{h}^{n} & =\boldsymbol{u}_{h}^{n}, \\
\hat{\boldsymbol{u}}_{h}^{n-1} & =\boldsymbol{u}_{h}^{n-1},
\end{aligned}
$$

and the intermediate velocity and pressure subscales $\hat{\tilde{\boldsymbol{u}}}^{n+1}$ and $\hat{\tilde{p}}^{n+1}$. The steps of the fractional step method are:

1. Convection-diffusion equation. We solve for the intermediate quantities $\hat{\boldsymbol{u}}_{h}^{n+1}, \hat{\tilde{\boldsymbol{u}}}^{n+1}$ and $\hat{\tilde{p}}^{n+1}$ :

$$
\begin{gathered}
\left(\boldsymbol{v}_{h}, \delta_{t} \hat{\boldsymbol{u}}_{h}^{n+1}\right)+B\left(\left[\boldsymbol{v}_{h}, 0\right],\left[\hat{\boldsymbol{u}}^{n+1} ; \hat{\boldsymbol{u}}_{h}^{n+1}, p_{h}^{n}\right]\right) \\
+\sum_{K}\left\langle-\nu \Delta \boldsymbol{v}_{h}-\hat{\boldsymbol{u}}^{n+1} \cdot \nabla \boldsymbol{v}_{h}, \hat{\tilde{\boldsymbol{u}}}^{n+1}\right\rangle_{K}-\sum_{K}\left\langle\nabla \cdot \boldsymbol{v}_{h}, \hat{\tilde{p}}^{n+1}\right\rangle_{K}=\left\langle\boldsymbol{v}_{h}, \boldsymbol{f}^{n+1}\right\rangle,
\end{gathered}
$$

where $\hat{\tilde{\boldsymbol{u}}}^{n+1}$ is the solution of:

$$
\hat{\tilde{\boldsymbol{u}}}^{n+1}=\phi_{\tilde{\boldsymbol{u}}}\left(\hat{\boldsymbol{u}}_{h}^{n+1}, p_{h}^{n}, t^{n+1}\right)
$$

as defined in (13) and $\hat{\tilde{p}}^{n+1}$ is the solution of:

$$
\hat{\tilde{p}}^{n+1}=\phi_{\tilde{p}}\left(\hat{\boldsymbol{u}}_{h}^{n+1}\right),
$$

as defined in (14).

2. Pressure Poisson equation. We solve for $p_{h}^{n+1}$ and $\tilde{\boldsymbol{u}}^{n+1}$ :

$$
\left(q_{h}, \nabla \cdot \hat{\boldsymbol{u}}_{h}^{n+1}\right)-\sum_{K}\left\langle\nabla q_{h}, \tilde{\boldsymbol{u}}^{n+1}\right\rangle_{K}=-\frac{2 \delta t}{3}\left(\nabla\left(p_{h}^{n+1}-p_{h}^{n}\right), \nabla q_{h}\right),
$$

where $\tilde{\boldsymbol{u}}^{n+1}$ is the solution of:

$$
\tilde{\boldsymbol{u}}^{n+1}=\phi_{\tilde{\boldsymbol{u}}}\left(\hat{\boldsymbol{u}}_{h}^{n+1}, p_{h}^{n+1}, t\right),
$$

as defined in (13). For the temporal integration of (23), we consider $\tilde{\boldsymbol{u}}^{n}=\hat{\tilde{\boldsymbol{u}}}^{n}$. In this way only the intermediate subscale velocity needs to be tracked in time.

3. Projection step: We solve for $\boldsymbol{u}_{h}^{n+1}$ :

$$
\frac{3}{2 \delta t}\left(\boldsymbol{v}_{h}, \boldsymbol{u}_{h}^{n+1}-\hat{\boldsymbol{u}}_{h}^{n+1}\right)+\left(\boldsymbol{v}_{h},\left(\nabla p_{h}^{n+1}-\nabla p_{h}^{n}\right)=0 .\right.
$$

\section{Heat transfer equation}

\subsection{Problem statement}

In this section we summarize the finite element approximation of the heat transfer equation using the OSS approach. Let us consider the transient heat transfer equation, which consists of finding $\theta: \Omega \times(0, T) \longrightarrow \mathbb{R}^{3}$ such that:

$$
\begin{aligned}
\partial_{t} \theta+\boldsymbol{u} \cdot \nabla \theta-\kappa \Delta \theta & =q & & \text { in } \Omega \\
\theta & =\bar{\theta} & & \text { on } \Gamma_{D \theta}, \\
\boldsymbol{n} \cdot \nabla \theta & =0 & & \text { on } \Gamma_{N \theta},
\end{aligned}
$$

where $\theta$ is the temperature field, $\kappa$ is the thermal diffusivity and $q$ is now the heat source term. Initial conditions have to be appended to this problem. 


\subsection{Finite element approximation}

Let us define $\Psi=H^{1}(\Omega)$, and the finite element space defined through the finite element partition $\Psi_{h} \subset \Psi$. The Galerkin finite element approximation of equation (24) consists of finding $\theta_{h} \in C^{1}\left(0, T ; \Psi_{h}\right)$ such that:

$$
\left(\psi_{h}, \partial_{t} \theta_{h}\right)+\left(\psi_{h}, \boldsymbol{u} \cdot \nabla \theta_{h}\right)+\kappa\left(\nabla \psi_{h}, \nabla \theta_{h}\right)=\left\langle\psi_{h}, q\right\rangle \quad \forall \psi_{h} \in \Psi_{h}
$$

with the corresponding initial and boundary conditions. Similarly to the Navier-Stokes problem, the finite element approximation (25) suffers from instability problems caused by the convective term. Stabilization is required, and again physically based numerical stabilization can be obtained by using the VMS method. Following a process analogous to the one presented in Section 2, the subscales can be modeled as a function of the finite element part of the temperature solution field, $\theta_{h}$ :

$$
\partial_{t} \tilde{\theta}+\tau_{\theta K}^{-1} \tilde{\theta}=P_{\tilde{\Psi}}\left(q-\left(\partial_{t} \theta_{h}-\kappa \Delta \theta_{h}+\boldsymbol{u} \cdot \nabla \theta_{h}\right)\right)
$$

with

$$
\tau_{\theta K}=\left(c_{\theta 1} \frac{\kappa}{h^{2}}+c_{\theta 2} \frac{|\boldsymbol{u}|}{h}\right)^{-1} .
$$

$c_{\theta 1}$ and $c_{\theta 2}$ are algorithmic constants, which in practice coincide with $c_{1}$ and $c_{2}$ in equation (10). Again, several choices are possible for the space of subgrid scales. As in the Navier-Stokes equations, we advocate for the use of Orthogonal Subgrid Scales, that is, we choose the space for temperature subscales $\tilde{\Psi}$ to be:

$$
\tilde{\Psi}=\Psi_{h}^{\perp} \cap \Psi .
$$

The stabilized finite element heat transfer problem is obtained by introducing the scale splitting in equation (25) and replacing the subscales by its approximation (26):

$$
\begin{gathered}
\left(\psi_{h}, \partial_{t} \theta_{h}\right)+\left(\psi_{h}, \boldsymbol{u} \cdot \nabla \theta_{h}\right)+\kappa\left(\nabla \psi_{h}, \nabla \theta_{h}\right) \\
+\sum_{K}\left\langle\psi_{h}, \partial_{t} \tilde{\theta}\right\rangle_{K}+\sum_{K}\left\langle-\kappa \Delta \psi_{h}-\boldsymbol{u} \cdot \nabla \psi_{h}, \tilde{\theta}\right\rangle_{K}=\left(\psi_{h}, q\right) \quad \forall \psi_{h} \in \Psi_{h} .
\end{gathered}
$$

\subsection{Turbulent thermal dissipation for the OSS heat transfer equation}

As done for the Navier-Stokes equations, we now deal with the numerical-turbulent dissipation of the OSS scheme for the heat transfer equation. We start by defining the thermal energy per unit volume in the computational domain $\Omega$ as:

$$
H=\frac{1}{2} \rho c_{p} \theta^{2}
$$

where $c_{p}$ is the heat capacity and $\rho$ the density of the fluid. The continuous thermal energy balance equation can be obtained by taking $\psi=\theta$ in equation (25), and multiplying the equation by $\rho c_{p}$. After some manipulations, and taking into account the incompressibility of $\boldsymbol{u}$, we obtain:

$$
\frac{\partial}{\partial t} \int_{\Omega} H+\int_{\partial \Omega} \boldsymbol{n} \cdot \boldsymbol{u} H=\left\langle\theta, \rho c_{p} q\right\rangle-\rho c_{p} \kappa(\nabla \theta, \nabla \theta) .
$$

In this case, the variation of thermal energy $\frac{\partial}{\partial t} \int_{\Omega} H$ in the computational domain is due to the convective flux of thermal energy through the domain boundary $\int_{\partial \Omega} \boldsymbol{n} \cdot \boldsymbol{u} H$, the increment of thermal energy caused by the heat source $\left\langle\theta, \rho c_{p} q\right\rangle$ and the decrement of thermal energy 
caused by the thermal dissipation $\chi=\rho c_{p} \kappa(\nabla \theta, \nabla \theta)$. The discrete counterpart of equation (28) is obtained by taking $\psi_{h}=\theta_{h}$ in equation (27):

$$
\begin{gathered}
\frac{\partial}{\partial t} \int_{\Omega} H_{h}+\int_{\partial \Omega} \boldsymbol{n} \cdot \boldsymbol{u} H_{h}=\left\langle\theta_{h}, \rho c_{p} q\right\rangle-\rho c_{p} \kappa\left(\nabla \theta_{h}, \nabla \theta_{h}\right) \\
-\sum_{K}\left\langle\rho c_{p} \theta_{h}, \partial_{t} \tilde{\theta}\right\rangle_{K}+\sum_{K}\left\langle\rho c_{p} \kappa \Delta \theta_{h}+\rho c_{p} \boldsymbol{u} \cdot \nabla \theta_{h}, \tilde{\theta}\right\rangle_{K},
\end{gathered}
$$

with:

$$
H_{h}=\frac{1}{2} \rho c_{p} \theta_{h}^{2}
$$

The two last terms in the right-hand side of equation (29) are called the thermal numerical dissipation:

$$
\begin{aligned}
& E_{\text {num }}=\sum_{K} \int_{K} \chi_{\text {num }} \\
& \chi_{\text {num }}=\rho c_{p} \theta_{h} \partial_{t} \tilde{\theta}-\left(\rho c_{p} \kappa \Delta \theta_{h}+\rho c_{p} \boldsymbol{u} \cdot \nabla \theta_{h}\right) \tilde{\theta} .
\end{aligned}
$$

$\chi_{\text {num }}$ appears due to the contribution of the stabilization terms and, as $\epsilon_{\text {num }}$ in the NavierStokes equations, is modeling the contribution to the dissipation due to the subscales. As a consequence, it can be considered as a model for the turbulent effects of the flow. Note that, contrary to other models, the turbulent Prandtl number which relates turbulent viscosity and turbulent thermal diffusivity is not required in this variational multiscale based turbulence model for the heat transfer equation. The total thermal dissipation is now modeled as:

$$
\chi=\chi_{h}+\chi_{\text {num }}
$$

with:

$$
\chi_{h}=\rho c_{p} \kappa\left|\nabla \theta_{h}\right|^{2}
$$

\subsection{Discretization in time}

The associated time discrete problem is: find $\theta_{h} \in \Psi_{h}$ for each $t \in[0, T]$ such that:

$$
\begin{array}{r}
\left(\psi_{h}, \delta_{t} \theta_{h}^{n+1}\right)+\left(\psi_{h}, \boldsymbol{u}^{n+1} \cdot \nabla \theta_{h}^{n+1}\right)+\kappa\left(\nabla \psi_{h}, \nabla \theta_{h}^{n+1}\right) \\
+\sum_{K}\left\langle\psi_{h}, \delta_{t} \tilde{\theta}^{n+1}\right\rangle_{K}+\sum_{K}\left\langle-\kappa \Delta \psi_{h}-\boldsymbol{u}^{n+1} \cdot \nabla \psi_{h}, \tilde{\theta}^{n+1}\right\rangle_{K}=\left(\psi_{h}, q\right) \quad \forall \psi_{h} \in \Psi_{h} .
\end{array}
$$

A second order backward difference scheme is used to approximate $\delta_{t} \theta_{h}^{n+1}$ and a first order backward Euler scheme is used to approximate the subscales derivative with respect to time, $\delta_{t} \tilde{\theta}^{n+1}$.

\section{Atmospheric seeing parameters}

In this section we describe the parameters which quantify the atmospheric seeing of a facility or observation site. We also relate them to the numerical approximations described in Sections 2 and 3. We focus especially on the relation with the turbulent dissipations $\epsilon_{\text {num }}$ and $\chi_{\text {num }}$ which appear in the stabilized discrete finite element equations due to the energy transfer to the non-resolved scales of the Kolmogorov cascade. 
The final optical parameters we aim to simulate numerically are the Fried parameter $r_{0}$ and the Greenwood frequency $f_{G}$. The Fried parameter is essential in adaptive optics. In the case of telescopes it allows to determine the number of segments into which a segmented mirror has to be split, or the distance between actuators for a continuous deformable mirror, by prescribing an admissible RMS distortion of a wavefront [2]. But the design of their actuators is also based on the so called Greenwood frequency, which is an indication of how fast the atmosphere is changing and defines the bandwidth of the servo control for an adaptive optics system (see [33] for more details). However, both parameters are a function of the integral along the optical path of light beams of the structure constant $C_{n}$ of the refractive index of a medium, $n(\boldsymbol{x}, t)$. See [13] for a detailed description of the relationship between $r_{0}, f_{G}$ and $C_{n}$.

This structure function can be related to the structure function of the temperature, the humidity and their joint structure parameter (see [32]). However, we will consider the humidity effects negligible. Thus, if we write the temperature dependence of $n$ as $n=n(\theta)$, we have

$$
C_{n}=\frac{\mathrm{d} n}{\mathrm{~d} \theta} C_{\theta},
$$

where $C_{\theta}$ is the structure function of the temperature. Assuming pressure equilibrium it is found that [34]

$$
C_{n}=\frac{79 \times 10^{-6}}{\bar{\theta}^{2}} \bar{p} C_{\theta},
$$

where $p$ is assumed to be measured in millibars and $\theta$ is the absolute temperature. Here and below, $\theta, p$ and $\boldsymbol{u}$ denote the solution of the continuous problems defined in equations (4) and (24) and the overbars denote mean quantities in the observation period.

In view of (34), the problem is to compute $C_{\theta}$. Once again in the inertial range of the Kolmogorov spectrum and assuming the temperature to be a passive quantity, it can be shown that (see [33])

$$
C_{\theta}^{2}=a^{2} \bar{\chi}_{\mathrm{m}} \bar{\epsilon}_{\mathrm{m}}^{-1 / 3}
$$

where $a$ is an empirical value called Obukhov-Corrsin constant (see [25, 36] for extensions and a discussion about Obukhov-Corrsin constants and on the validity of this approximation). In (35), $\bar{\chi}_{\mathrm{m}}$ denotes the mean molecular thermal diffusive dissipation and $\bar{\epsilon}_{\mathrm{m}}$ the mean molecular dissipation of kinetic energy of the flow. These parameters are given by

$$
\bar{\chi}_{\mathrm{m}}:=\rho c_{p} \kappa \overline{|\nabla \theta|^{2}}, \quad \bar{\epsilon}_{\mathrm{m}}:=2 \mu \overline{\left|\nabla^{s} \boldsymbol{u}\right|^{2}} .
$$

The problem is now closed: using (36) in (35) and the result back in (34) we have an expression to compute $C_{n}$ in terms of the flow variables $\boldsymbol{u}, p, \theta$ at each point.

Some questions remain open when we want to numerically apply the previous approximations for $C_{n}^{2}$ and $C_{\theta}^{2}$. The first issue is that, instead of working with $\boldsymbol{u}, p$, and $\theta$, we are going to be working with $\boldsymbol{u}_{h}, p_{h}$, and $\theta_{h}$, and a model for the velocity and temperature under-resolved scales $\tilde{\boldsymbol{u}}$ and $\tilde{\theta}$ given by equations (12) and (26). The first point to consider is the relation between the averaged quantities $\overline{\boldsymbol{u}}, \bar{p}$, and $\bar{\theta}$, and their finite element approximations. To this end we will recall that filtered unknowns in LES models need to maintain the mean of the original variables. Due to the close relationship of the presented VMS turbulence model to LES filtering, we will assume this to be true also for the finite element variables $\boldsymbol{u}_{h}, p_{h}$, and $\theta_{h}$. This means that we will consider

$$
\overline{\boldsymbol{u}} \approx \overline{\boldsymbol{u}}_{h}, \quad \bar{p} \approx \bar{p}_{h}, \quad \bar{\theta} \approx \bar{\theta}_{h} .
$$


The second point is how to compute the average kinetic and thermal energy dissipations $\bar{\epsilon}_{m}$ and $\bar{\chi}_{m}$. In order to do this we will make use of the definitions in equations (21) and (32), and we will compute the averaged dissipations as

$$
\bar{\epsilon}=\bar{\epsilon}_{h}+\bar{\epsilon}_{\text {num }}, \quad \bar{\chi}=\bar{\chi}_{h}+\bar{\chi}_{\text {num }} .
$$

We assume that $\bar{\epsilon} \approx \bar{\epsilon}_{\mathrm{m}}, \bar{\chi} \approx \bar{\chi}_{\mathrm{m}}$, that is, the model accounts properly for the molecular dissipation. This is proved in particular in [24] in a simplified setting. That the mean dissipation approximates the molecular dissipation is the general assumption of LES models.

Using approximations (37) and (38) in (35) and inserting the result in (34) it is found that:

$$
C_{n}=79 \times 10^{-6} \bar{\theta}_{h}^{-2} \bar{p}_{h} a \bar{\chi}^{1 / 2} \bar{\epsilon}^{-1 / 6} .
$$

Equation (39) is the expression we were looking for. It allows us to compute the structure function of the refractive index in terms of the flow variables resulting from a VMS numerical simulation.

\section{$5 \quad$ Numerical examples}

\subsection{Convective boundary layer}

The first numerical example consists of a convective boundary layer. This example was first presented in [8] where a method for the estimation of atmospheric seeing using the Dutch Atmospheric LES method (DALES) is presented. We use it here to compare the optical parameters obtained by using the VMS based dissipation model against the ones obtained using the Smagorinsky based dissipation model presented in [13], and a dissipation model based on the WALE subgrid scale model. The simulation domain is a parallelepiped whose base is a square with a $10 \mathrm{~km}$ side and a height of $2 \mathrm{~km}$. Air flow is caused by the presence of a heat flux through the inferior base $\left(0.1 \mathrm{~K} \mathrm{~m} \mathrm{~s}^{-1}\right)$ and an horizontal body acceleration term $\left(9.4325 \cdot 10^{-5}\right.$ $\mathrm{m} \mathrm{s}^{-2}$ ). Boundary conditions are periodic in the lateral boundaries and no fluid is allowed to trespass the superior and inferior boundaries of the domain. Also a wall law boundary condition [4] is used in the inferior boundary, which causes the presence of the boundary layer for this example. Initial conditions are given by a vertical temperature gradient set to $3 \mathrm{~K} \mathrm{~km}^{-1}$ and air at rest. The numerical simulation is run for 10000 seconds, with a time step of 1 second. The finite element mesh is a uniform structured tetrahedra mesh totaling 1.8 million elements. The mesh resolution is $150 \mathrm{~m}$ horizontally and $150 \mathrm{~m}$ vertically.

Fig. 1 shows the averaged temperature and velocity profiles along the vertical dimension. In the temperature profile the expected increase in mean temperature due to the heat flux in the inferior boundary is observed. At a greater height, the temperature values get closer to the initial condition temperature profile. Regarding the horizontal velocity profile, a boundary layer behavior is recovered with a large velocity gradient close to the floor. Moreover, a slight decrease in the average velocity is observed in the region where the temperature joins the initial constant vertical gradient (1000 m height). Fig. 2 shows a snapshot of the velocity and temperature fields at the end of the simulation. The velocity snapshot allows to see how hot air in the ground tends to be convected up due to the Boussinesq forces. The temperature field, on the other hand, is smooth, large temperature gradients appear only in the ground where the heat flux causes bubbles of hot air to appear. Finally Fig. 3 shows a plot of the dissipation values for the kinetic energy balance at the end of the simulation obtained using the OSS Variational Multiscale Method. The top plot corresponds to dissipation values computed at 
numerical integration points and its extrapolation to the nodes of each element. Elements with a partially white area correspond to elements where the dissipation at some of the numerical integration points is negative (or the dissipation extrapolated to the nodes of the element is negative). In the bottom plot the nodally averaged (through a lumped $L^{2}$ projection) is shown. The nodally averaged dissipation values are positive everywhere. This is in agreement with the expected behavior for the modeling of backscatter, where dissipation values can be locally negative (accounting for the transmission of energy from the small scales to the large scales), but need to be positive when averaged in space and time.

Fig. 4 shows a comparison of the $C_{n}^{2}$ fields obtained by using the OSS based model for the computation of the viscous and thermal dissipations and the structure constants, the Smagorinsky and the WALE models. It can be observed that the three models yield qualitatively similar results: the magnitude of the structure constant $C_{n}^{2}$ is large close to the ground, where large temperature gradients exist due to the thermal heat flux. After the first few meters, the $C_{n}^{2}$ value starts to diminish with height (as the cyan regions denote), although the $C_{n}^{2}$ coefficient magnitude is larger in those regions where there is a hot air bubble moving up (vertical yellow patches close to the ground). In the mid-height region of Fig. 4 (corresponding to a height of $1000 \mathrm{~m}$ ) the hot bubbles disappear due to the effect of the dominant horizontal flow. In this region, an increase of the $C_{n}^{2}$ values is observed. At a height of $1500 \mathrm{~m}$, the $C_{n}^{2}$ values diminish again. Due to the non-trespassing boundary condition, an spurious increase of the $C_{n}^{2}$ values is observed in the top wall. This increase appears due to the fact that large gradients of the velocity are obtained in this top non-trespassing boundary condition, and would not be there in a real open-flow boundary condition (which we cannot reproduce at the numerical level). It must also be noted that the $C_{n}^{2}$ field is smoother for the Smagorinsky and WALE models than for the OSS model. This is probably caused by the fact that the Smagorinsky and WALE models for the dissipations are based on the gradients of the recovered fields, while the dissipations for the OSS model are based on the component of the residual orthogonal to the finite element space, which is undoubtedly less smooth. Finally, Fig. 5 shows a comparison of the horizontally averaged $C_{n}^{2}$ values at the end of the simulation, including the results of [8] using the DALES model. It is clear that the presented results and the results in [8] are qualitatively similar. However, the increase in the $C_{n}^{2}$ at mid-height occurs at a lower height in the results from [8]. The largest differences are obtained in the region close to the ground. This can be caused by the fact that the $C_{n}^{2}$ parameter is very sensitive to small variations in the temperature gradients. The bottom region presents the largest temperature gradients, and small deviations in the computed temperature gradients can cause large variations in the obtained $C_{n}^{2}$ values. In spite of this, the results presented here are in good agreement with the results in [8], since, in most of the height (except for the bottom region very close to the ground), they lay within one standard deviation of the mean of the distribution of results statistically collected in [8].

This results allow to state that the $C_{n}^{2}$ values obtained through the Orthogonal Subgrid Scale model show a distribution which is qualitatively the same as the one obtained through physically based LES models (Smagorinsky, WALE and the DALES model in [8]). Since the $C_{n}^{2}$ values depend on the turbulent dissipation of the model, it means that the viscous and thermal turbulent dissipations obtained in the OSS method are qualitatively the same as the ones in the physically based LES models. This is remarkable, because the expressions for these dissipations were motivated exclusively by numerical arguments in the case of OSS, which contrasts with the physically based arguments used to derive the LES turbulent dissipations. 

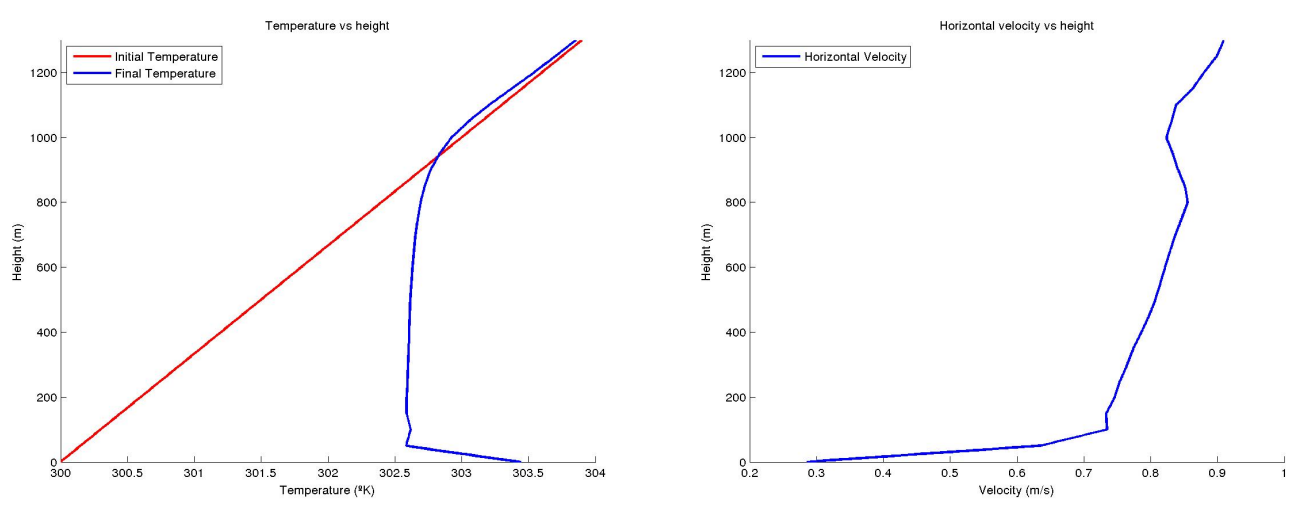

Figure 1: Temperature (left) and horizontal velocity (right) averaged profiles

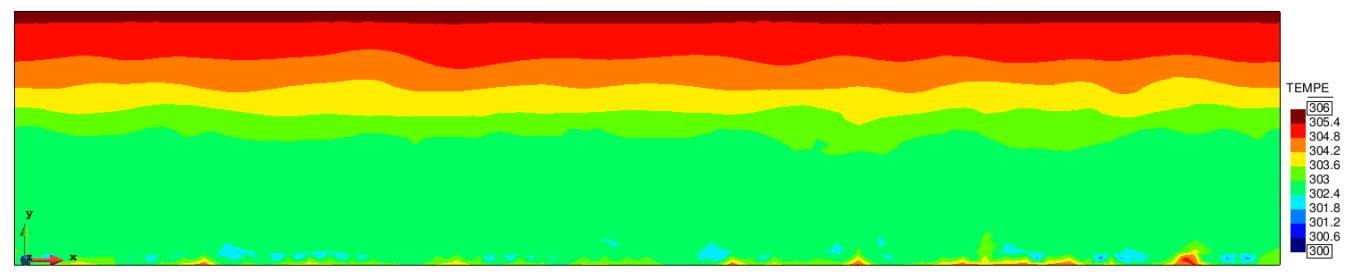

Gii

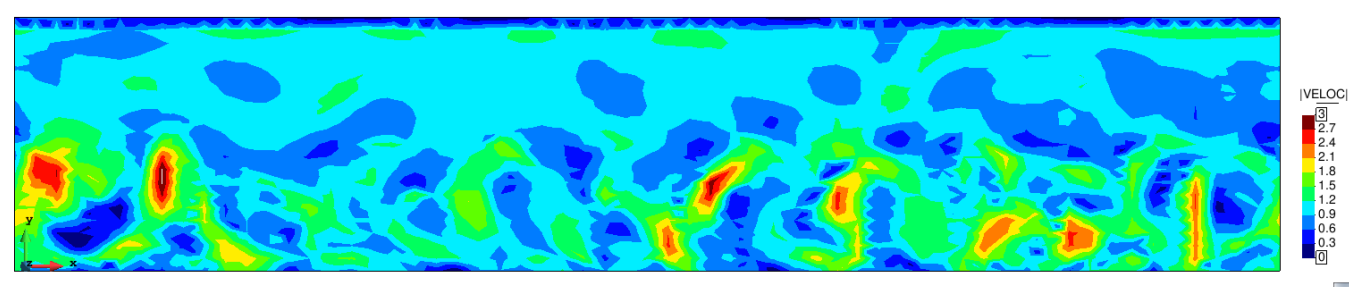

Gip

Figure 2: Temperature in ${ }^{\circ} \mathrm{K}$ (top) and Velocity (bottom) snapshots at the end of the simulation 


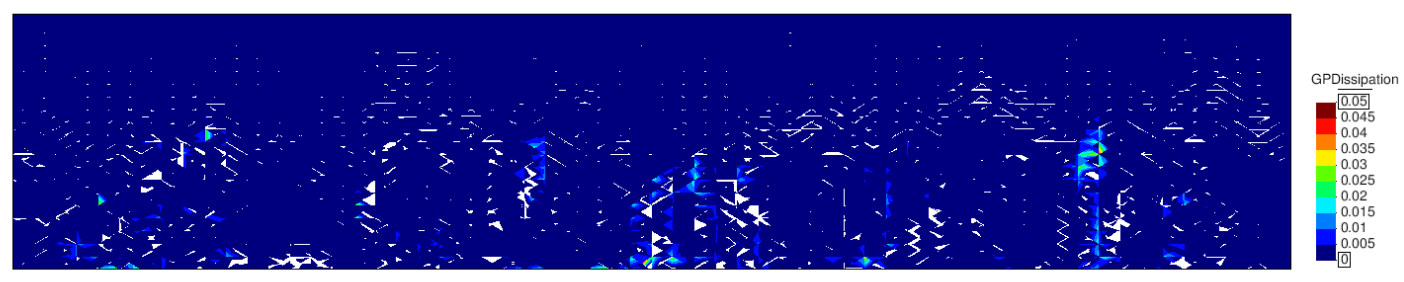

GiD

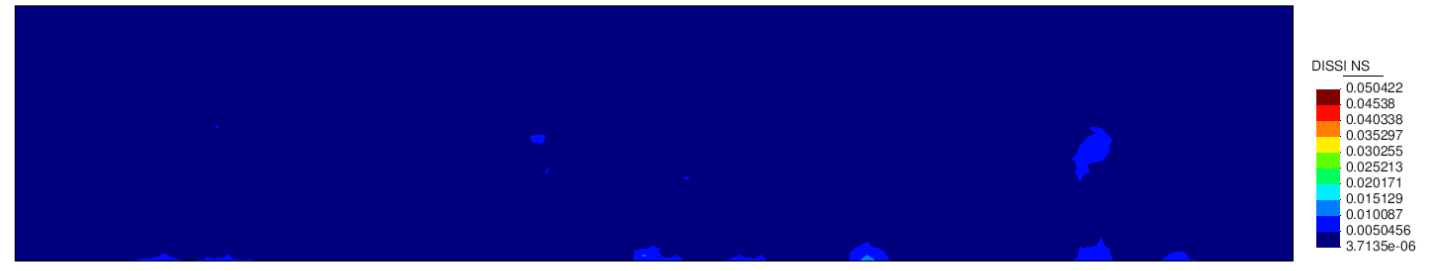

GiD

Figure 3: Dissipation values for the kinetic energy balance: numerical integration points (top) and nodally averaged (bottom) values.
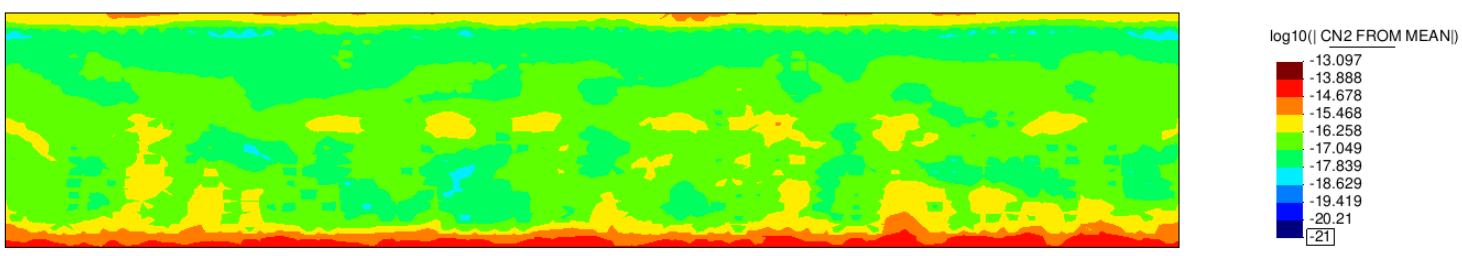

GiD
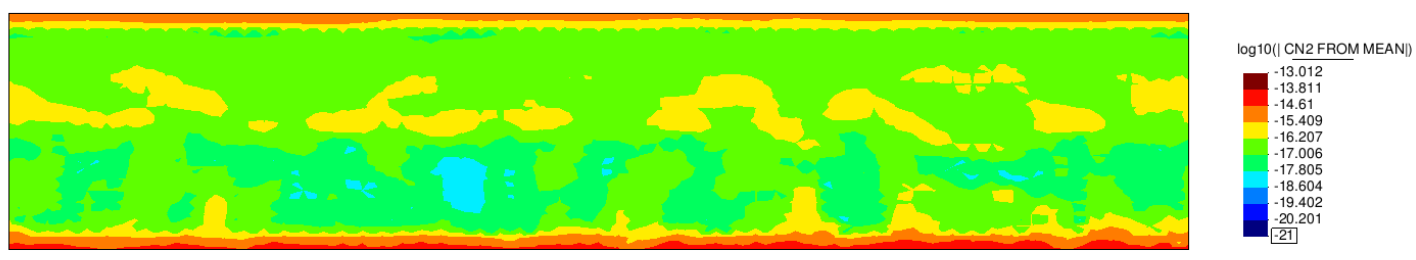

GiD

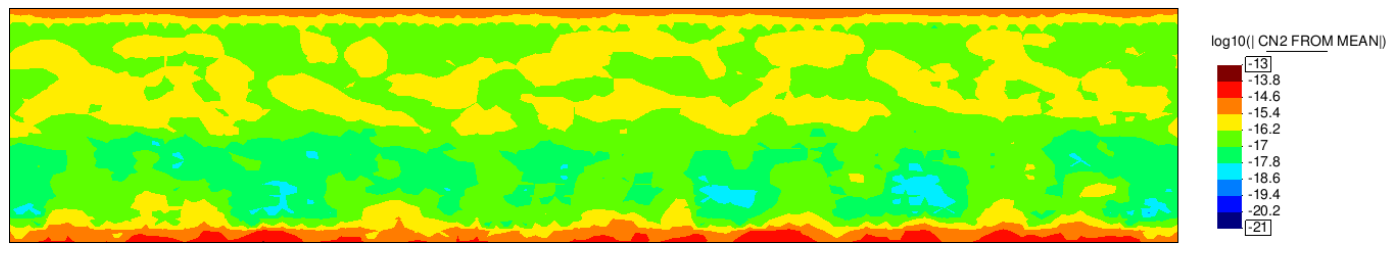

GiD

Figure 4: $C_{n}^{2}$ snapshots at the end of the simulation. From top to bottom: Variational Multiscale, Smagorinsky, WALE model. 


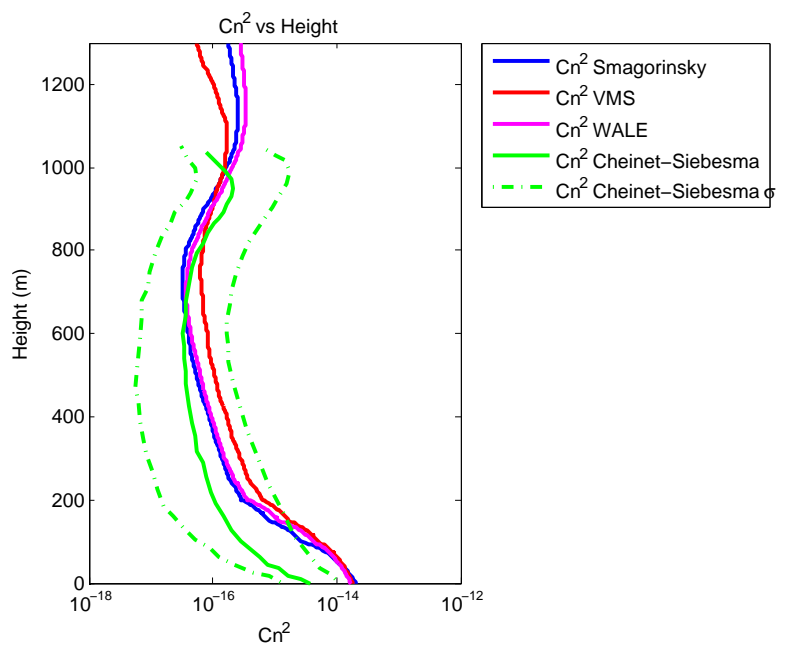

Figure 5: Averaged (horizontally) $C_{n}^{2}$ values at the end of the simulation. Comparison with the results in [8]. The green dotted lines represent the values which lie within one standard deviation in the statistical data collected in [8].

\subsection{Transfer optics and Coudé room}

The second example corresponds to a transfer optics chamber and Coudé room. The geometry and boundary conditions for this case were provided by the Astrophysical Institute of the Canary Islands during the design phase of the European Solar Telescope (EST). Fig. 6 shows the geometry and boundary conditions of the case. The spheres in the plot represent concentrated heat loads, which consist of $300 \mathrm{~mm} \varnothing$ and provide a heat source of $2 \mathrm{~W}\left(7.0738 \mathrm{~W} / \mathrm{m}^{2}\right)$. The thermal control system consists of air plenums blowing air at ambient temperature $\left(20^{\circ} \mathrm{C}\right)$, vertical velocity equal to $1 \mathrm{~m} / \mathrm{s}$, in the vertical direction from the top of the chamber (plenum area $=22.1 \mathrm{~m}^{2}$ ). Return air plenums are placed at the lower part of the chamber (plenum area $=314.16 \mathrm{~m}^{2}$ ). The objective of this case is to analyze the seeing degradation in the transfer optics chamber.

Velocity boundary conditions in the lateral walls and the heating spheres correspond to a wall-law condition. Inflow velocity is set to $1 \mathrm{~m} / \mathrm{s}$, and outflow velocity is left free. The numerical simulation is run for 100 seconds, after which the flow is considered to be completely developed. The time step is set to 0.2 seconds. The finite element mesh is composed of 3.4 million tetrahedra, with a local refinement in the regions close to the heating spheres (element size equal to $0.03 \mathrm{~m}$ ) and larger elements in in the regions far from them (element size equal to $0.3 \mathrm{~m})$.

Fig. 7 shows a velocity and temperature snapshot after the flow has been completely developed. The velocity is larger in the central region, where most of the injected air flow is circulating. The heating spheres oppose to the flow, which causes some boundary layers to appear. This will have a negative effect in the resulting seeing conditions. Regarding the temperature, the largest temperatures and temperature gradients are found on the surface of the heating spheres, and hot air jets are found following the path of the vertical air inflow.

Fig. 8 shows a comparison of the $C_{n}^{2}$ values in the transfer optics room, obtained by using 


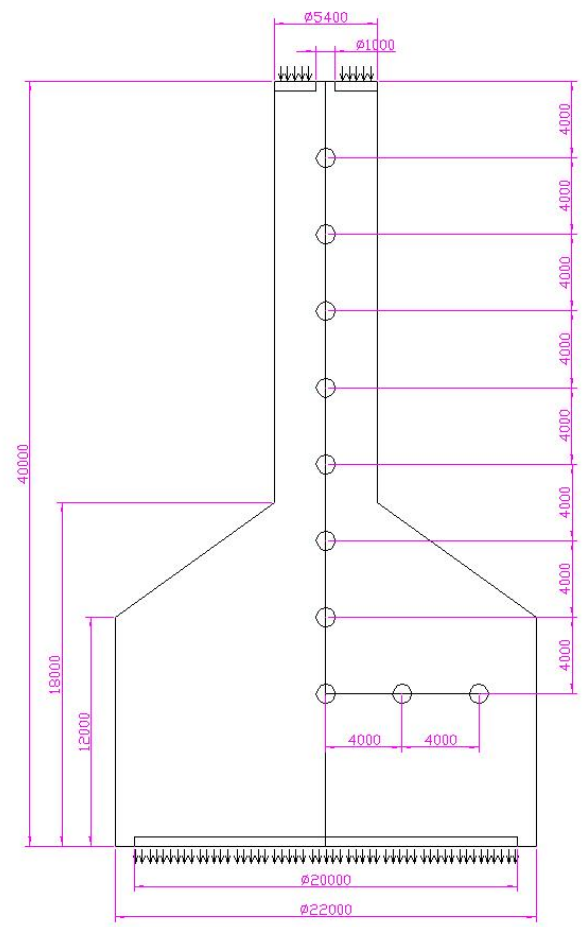

Figure 6: Geometry of the transfer optics and Coudé room (distances in mm)
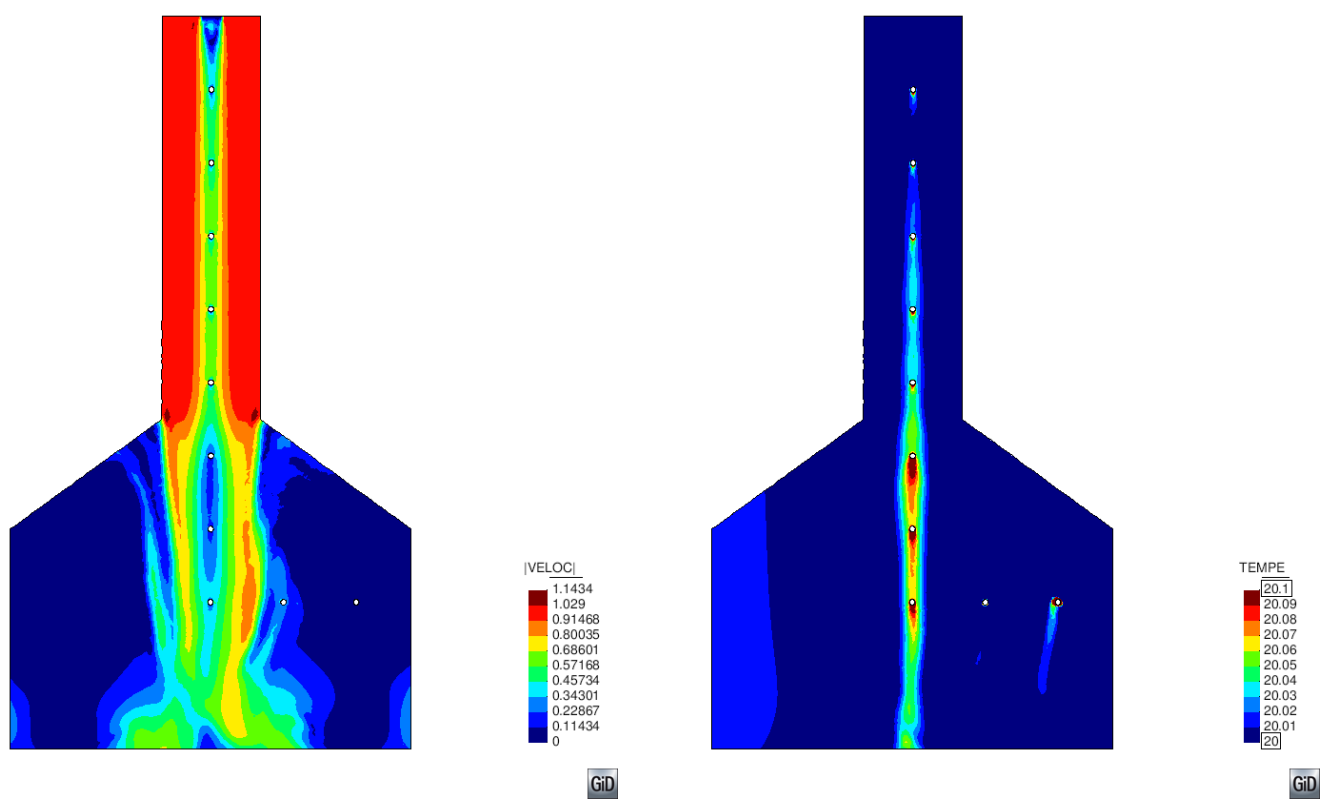

Figure 7: Velocity (left) and temperature in ${ }^{\circ} \mathrm{C}$ (right) snapshots at the transfer optics and Coudé room. 
the OSS, the Smagorinsky and the WALE models. It is clear that these models provide very similar results in this case, the $C_{n}^{2}$ field being less smooth for the OSS case.

\subsection{Flow around a telescope enclosure}

In the last example we simulate the atmospheric seeing around a telescope enclosure. In particular, this corresponds to one of the tentative designs of the European - Extremely Large Telescope (E-ELT) enclosure. Fig. 9 shows the geometry of the enclosure. The objective of this simulation was to evaluate the effect of a frontal wind shield in the atmospheric seeing. The enclosure has 4 meter sized windows which facilitate the natural ventilation of the building. The enclosure diameter is $85 \mathrm{~m}$, with a maximum height of $79 \mathrm{~m}$. The external domain for the simulation is a $600 \times 600 \times 1500 \mathrm{~m}^{3}$ box. The finite element mesh is composed of 3.3 million tetrahedra, with element sizes ranging from $1 \mathrm{~m}$ (close to the telescope) to $20 \mathrm{~m}$ (in the open flow region).

A wall-law velocity boundary condition [4] is set on the ground and building surfaces, while a non-trespassing boundary condition is set on the lateral walls of the bounding box. The inflow velocity is set to $1 \mathrm{~m} / \mathrm{s}$. Regarding temperature boundary conditions, we impose the following expression for temperature as a function of height:

$$
\theta=12-0.7 \ln \left(\frac{z+0.01}{0.02}\right)
$$

where the height $z$ is expressed in meters and the resulting temperature is expressed in ${ }^{\circ} \mathrm{C}$. The initial temperature profile is depicted in Fig. 10.

Fig. 11 shows the velocity and temperature fields at a cut along the stream-wise direction after the flow has been fully developed. Turbulent vortexes appear behind the telescope, the largest velocity gradients occurring on the surface of the telescope. The maximum absolute value velocity appears after the flow has been detached from the telescope enclosure, and a low-speed recirculation zone can be observed leeward from the telescope. Regarding the temperature field, the maximum temperature and temperature gradients are again found on the surface of the telescope. The interior of the telescope shows larger temperatures when compared to the exterior domain, despite the large ventilation windows put in place in order to minimize temperature gradients. Intermediate temperature values are also found in the recirculation area behind the telescope.

Fig. 12 shows a comparison of the $C_{n}^{2}$ profiles obtained by using the OSS, Smagorinsky and WALE dissipation models. As in the previous cases, maximum values for the $C_{n}^{2}$ parameter are found in the regions where the velocity and temperature gradients are larger, which coincide with the terrain ground, the telescope enclosure surface and the area where the detachment of the flow occurs. The low speed recirculation zone behind the telescope also shows large values for the $C_{n}^{2}$ parameter, which is due to the large temperature gradients. If we compare the results of the various models, results are again qualitatively equivalent, the results being smoother for the Smagorinsky and WALE models.

Let us say again that this agreement is remarkable if we take into account that the expressions for the calculation of dissipations were motivated by physical arguments in the case of the LES models and, on the contrary, by numerical arguments in the case of the OSS variational multiscale method. 

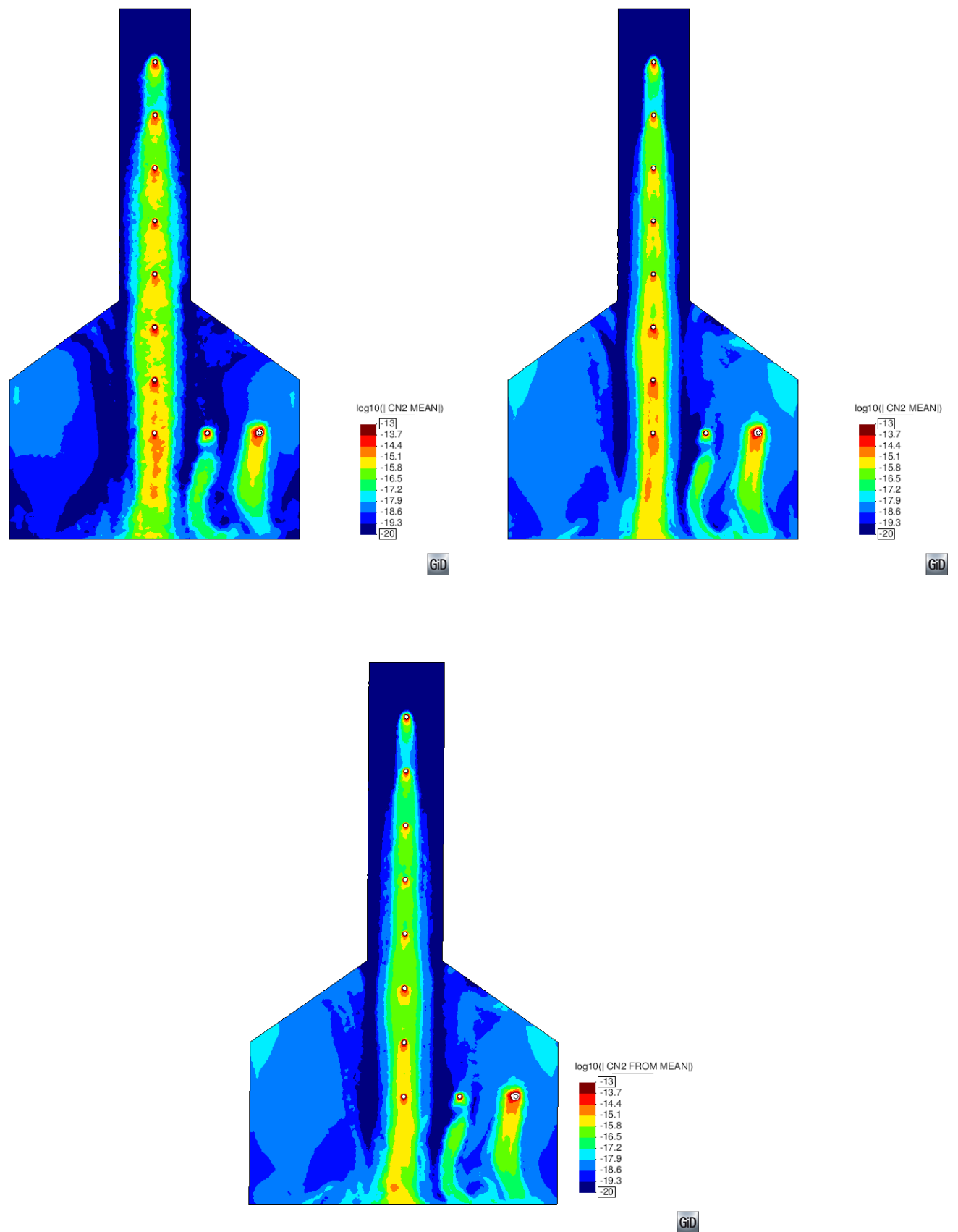

GiD

Figure 8: $C_{n}^{2}$ snapshots for the Coudé room. Variational Multiscale (top left), Smagorinsky (top right) and WALE (bottom) models. 


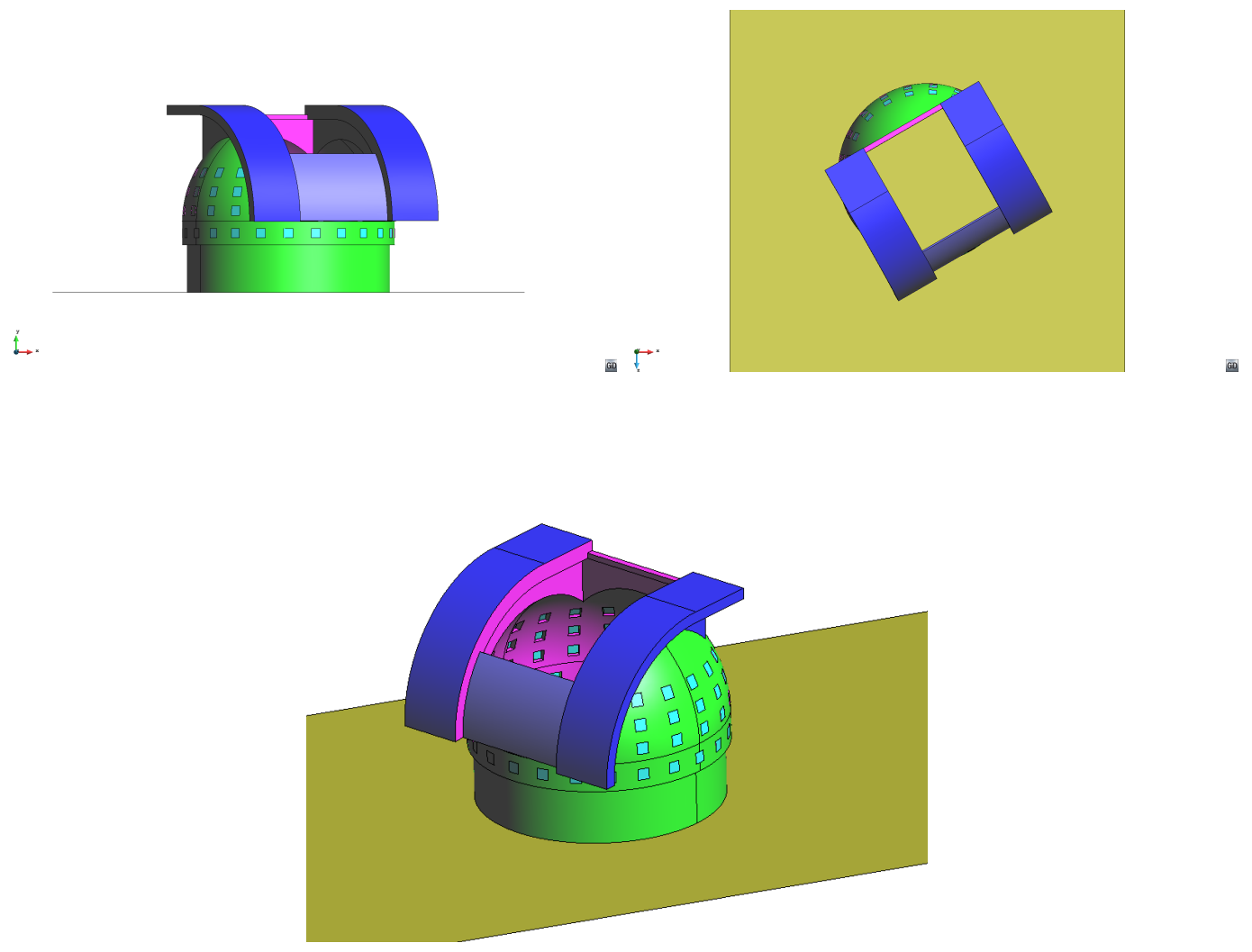

Figure 9: External geometry for the E-ELT enclosure. Front, top and perspective views.

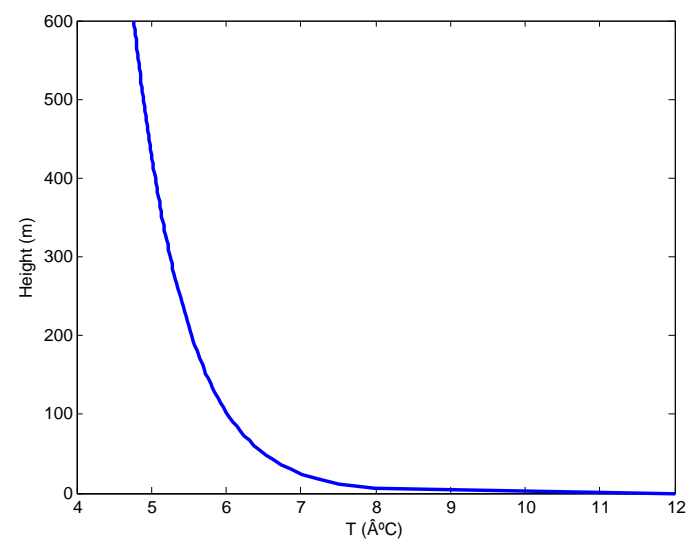

Figure 10: Temperature inflow boundary condition as a function of height 


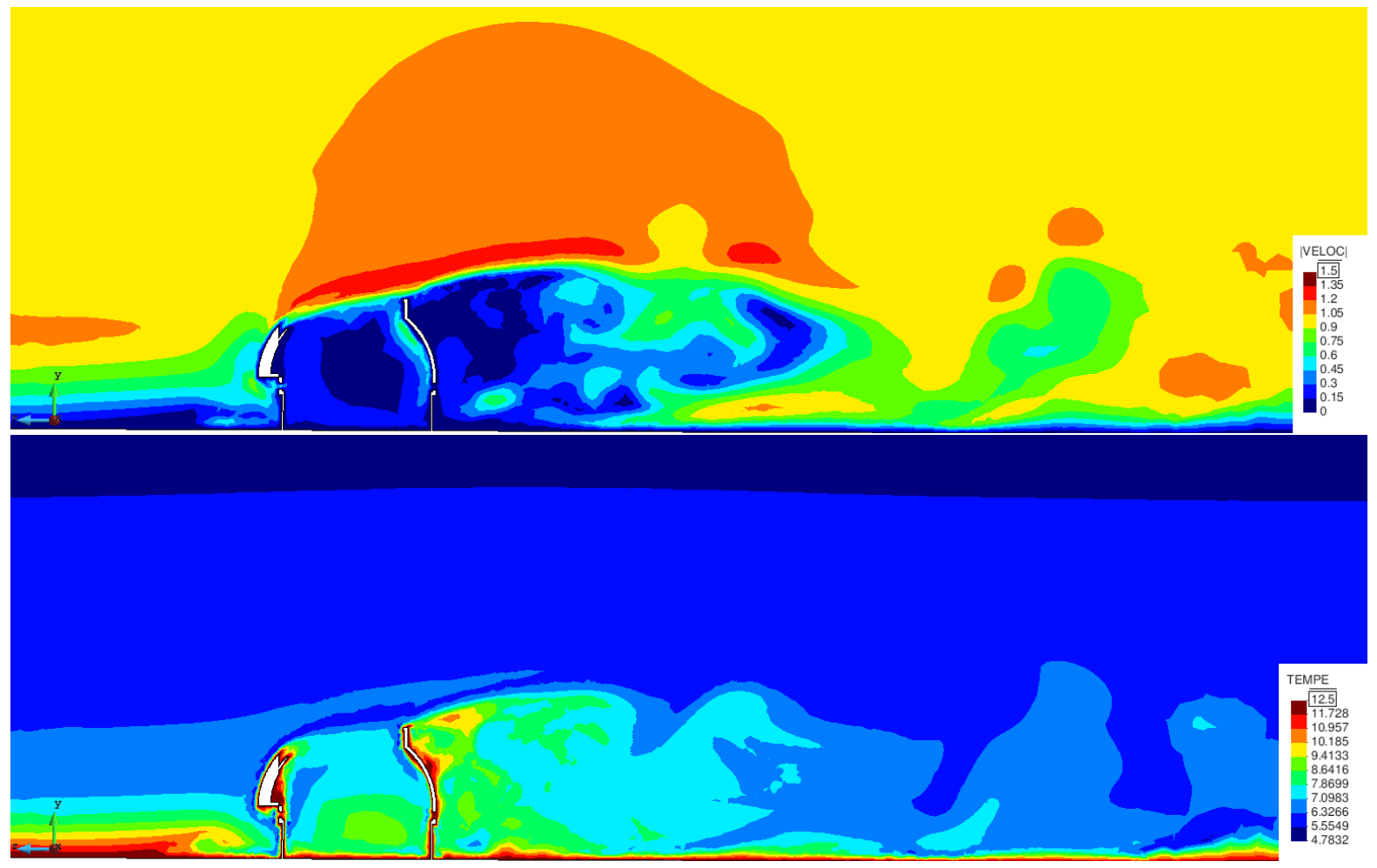

Figure 11: Velocity and temperature fields for the flow around a telescope enclosure

\section{Conclusions}

In this work we have presented a numerical model for the estimation of atmospheric seeing in observation sites. The main feature of the proposed model is that it is based on the numerical dissipations which arise from a particular version of the Variational Multiscale Method, the Orthogonal Subgrid Scale method. The advantage of using this kind of models relies on the fact that, by decomposing the fields of interest into coarse and fine scales, they are able to deal simultaneously with the sources of numerical instabilities and the modeling of turbulent effects. In the present work we have summarized the properties of our variational multiscale method, which is based on modeling the numerical subscales in an as complete as possible manner: the subscales are considered to be transient in time, non-linear, and orthogonal to the finite element space. This leads not only to the resolution of numerical stability issues (advection and the use of arbitrary interpolations for velocity and pressure), but also to a rich representation of turbulent phenomena. Based on this turbulence model, we have developed the expressions for the viscous and thermal dissipations, $\epsilon_{\text {num }}$ and $\chi_{\text {num }}$, which have been used for evaluating the constant of structure of the refraction index $C_{n}^{2}$ following the classical model developed by Tatarski.

In the numerical examples section we have tested the performance of the method in three practical cases, namely a convective boundary layer, the flow inside a transfer optics room, and the flow around a telescope enclosure. In all three cases we have compared our model with the results obtained by using a Smagorinsky and WALE models for evaluating the viscous and thermal dissipations, and, in the convective boundary layer case, with the results presented in [8]. The numerical examples show that the method is capable of doing an accurate estimation of the $C_{n}^{2}$ coefficients. This fact does not only provide us with a new numerical tool for the 


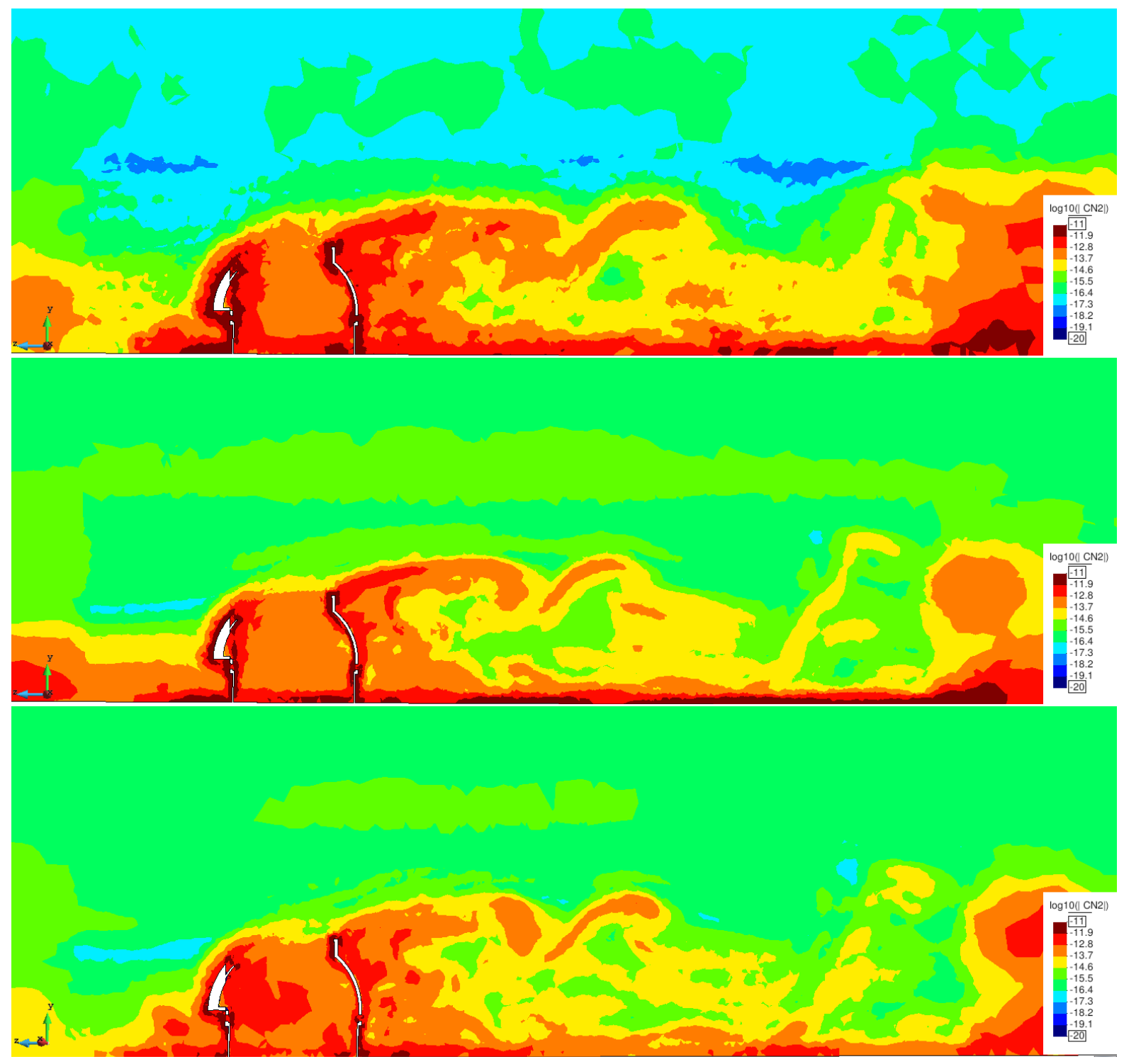

Figure 12: $C_{n}^{2}$ field for the flow around a telescope enclosure. From top to bottom: Variational Multiscale, Smagorinsky and WALE models. 
evaluation of the atmospheric seeing but it also adds arguments in favor of the viability of implicit LES methods which rely on the numerical stabilization mechanisms for the modeling of turbulence.

\section{ACKNOWLEDGEMENTS}

This work was partially supported by the European Research Council under the SOLARNET research network FP7 - 312495, and partially supported by the Ministerio de Economía y Competitividad of the Spanish Government under the Plan Nacional de Investigación 2012: AYA2012-33490. Joan Baiges gratefully acknowledges the support received from the MICINN of the Spanish Government through a "Juan de la Cierva" Postdoctoral grant. Ramon Codina gratefully acknowledges the support received from the Catalan Government through the ICREA Acadèmia Research Program.

\section{References}

[1] R.J. Alliss and B.D. Felton. Validation of optical turbulence simulations from a numerical weather prediction model in support of adaptive optics design. In Advanced Maui Optical and Space Surveillance Technologies Conference, volume 1, page 54, 2009.

[2] J.M. Beckers. Adaptive optics for astronomy: Principles, Perfomance and Applications. Annual Reviews in Astronomy and Astrophysics, 1993.

[3] FCM Bettonvil, R Codina, RH Hammerschlag, APL Jägers, JNM Kommers, SJ Van Leverink, G Sliepen, and S Visser. The enclosure for the European Solar Telescope (EST). In SPIE Astronomical Telescopes and Instrumentation: Observational Frontiers of Astronomy for the New Decade, pages 773333-773333. International Society for Optics and Photonics, 2010.

[4] Peter Bradshaw and George P Huang. The law of the wall in turbulent flow. Proceedings of the Royal Society of London. Series A: Mathematical and Physical Sciences, 451(1941):165-188, 1995.

[5] L Cavaller, M Collados, and J Castro. Performance simulations for the conceptual design of the European Solar Telescope (EST). In Proc. of SPIE Vol, volume 8336, pages 83360G-1, 2011.

[6] S. Cheinet, A. Beljaars, K. Weiss-Wrana, and Y. Hurtaud. The use of weather forecasts to characterise near-surface optical turbulence. Boundary-Layer Meteorology, 138:453-473, 2011.

[7] S. Cheinet and A. P. Siebesma. The impact of boundary layer turbulence on optical propagation. In Optics in Atmospheric Propagation and Adaptive Systems X. Proc. of SPIE Vol. 6747, pages $1-12,2007$.

[8] S. Cheinet and A. P. Siebesma. Variability of local structure parameters in the convective boundary layer. J. Atmos. Sci., 66:1002-1017, 2009.

[9] T. Cherubini and S. Businger. An operational perspective for modeling optical turbulence, in Seeing Clearly: The impact of Atmospheric Turbulence on the Propagation of Extraterrestrial Radiation, T. Cherubini and S. Businger (Eds.). VBW Publishing, 2010.

[10] T. Cherubini, S. Businger, and R. Lyman. Modeling optical turbulence and seeing over Mauna Kea: Verification and algorithm refinement. J. Appl. Meteor. Climatol., 47:3033-3043, 2008.

[11] R. Codina. A stabilized finite element method for generalized stationary incompressible flows. Computer Methods in Applied Mechanics and Engineering, 190:2681-2706, 2001.

[12] R. Codina. Stabilized finite element approximation of transient incompressible flows using orthogonal subscales. Computer Methods in Applied Mechanics and Engineering, 191:4295-4321, 2002.

[13] R. Codina, J. Baiges, D. Pérez-Sánchez, and M. Collados. A numerical strategy to compute optical parameters in turbulent flow: Application to telescopes. Computers $\&$ Fluids, 39(1):87 - 98, 2010. 
[14] R. Codina and J. Principe. Dynamic subscales in the finite element approximation of thermally coupled incompressible flows. International Journal for Numerical Methods in Fluids, 54:707-730, 2007.

[15] R. Codina, J. Principe, and M. Ávila. Finite element approximation of turbulent thermally coupled incompressible flows with numerical sub-grid scale modelling. International Journal of Numerical Methods for Heat \&5 Fluid Flow, 20(5):492-516, 2010.

[16] R. Codina, J. Principe, and S. Badia. Dissipative structure and long term behavior of a finite element approximation of incompressible flows with numerical subgrid scale modeling. In Multiscale Methods in Computational Mechanics, pages 75-93. Springer, 2011.

[17] R. Codina, J. Principe, and J. Baiges. Subscales on the element boundaries in the variational twoscale finite element method. Computer Methods in Applied Mechanics and Engineering, 198:838$852,2009$.

[18] R. Codina, J. Principe, O. Guasch, and S. Badia. Time dependent subscales in the stabilized finite element approximation of incompressible flow problems. Computer Methods in Applied Mechanics and Engineering, 196:2413-2430, 2007.

[19] O. Colomés, S. Badia, R. Codina, and J. Principe. Assessment of variational multiscale models for the large eddy simulation of turbulent incompressible flows. Submitted.

[20] A.F. de Baas and M. Sarazin. The temperature structure fuction for a complex terrain. Eighth Symposium on turbulent shear flows., pages 1-6, Munich September 9-11, 1991.

[21] R. Frehlich, R. Sharman, F. Vandenberghe, W. Yu, Y. Liu, J. Knievel, and G. Jumper. Estimates of $\mathrm{Cn} 2$ from Numerical Weather Prediction Model Output and Comparison with Thermosonde Data. Journal of Applied Meteorology and Climatology, 49(8):1742-1755, 2010.

[22] C Giordano, J Vernin, H Vázquez Ramió, C Muñoz-Tuñón, AM Varela, and H Trinquet. Atmospheric and seeing forecast: Wrf model validation with in situ measurements at orm. Monthly Notices of the Royal Astronomical Society, 430(4):3102-3111, 2013.

[23] V. Gravemeier. The variational mulstiscale method for laminar and turbulent flow. Archives of Computational Mechanics-State of the Art Reviews, 13:249-324, 2006.

[24] O. Guasch and R. Codina. Statistical behavior of the orthogonal subgrid scale stabilization terms in the finite element large eddy simulation of turbulent flows. Computer Methods in Applied Mechanics and Engineering, 261:154-166, 2013.

[25] R.J. Hill. Structure functions and spectra of scalar quantities in the inertial-convective and viscousconvective ranges of turbulence. Journal of the atmospheric sciences, 46:2245-2251, 1989.

[26] T.J.R. Hughes, G.R. Feijóo, L. Mazzei, and J.B. Quincy. The variational multiscale method-a paradigm for computational mechanics. Computer Methods in Applied Mechanics and Engineering, $166: 3-24,1998$.

[27] T.J.R. Hughes, L. Mazzei, and K.E. Jansen. Large eddy simulation and the variational multiscale method. Computing and Visualization in Science, 3:47-59, 2000.

[28] S.L. Keil, T.R. Rimmele, and J. Wagner. Advanced technology solar telescope. Earth, Moon, and Planets, 104(1-4):77-82, 2009.

[29] E. Masciadri, J. Vernin, and P. Bougeault. 3D mapping of optical turbulence using an atmospheric numerical model. Part I. Astron. Astrophys. Suppl. Ser., 137:185-202, 1999.

[30] E. Masciadri, J. Vernin, and P. Bougeault. 3D mapping of optical turbulence using an atmospheric numerical model. Part II. Astron. Astrophys. Suppl. Ser., 137:203-216, 1999.

[31] E. Masciadri, J. Vernin, and P. Bougeault. 3D numerical simulations of optical turbulence at the Roque de Los Muchachos Observatory using the atmospherical model Meso-Nh. Astronomy \& Astrophysics, 365:699-708, 2001. 
[32] L.J. Peltier and J.C. Wyngaard. Structure function parameters in the convective boundary layer from large eddy simulation. Journal of the atmospheric sciences, 52:3641-3660, 1995.

[33] F. Roddier. Adaptive optics in astronomy. Cambridge University Press, 2004.

[34] V.I. Tatarski. Wave propagation in a turbulent medium. Dover Publications, INC, 1961.

[35] H. Trinquet and J. Vernin. A statistical model to forecast the profile of the index structure constant. Environ Fluid Mech, 7:397-407, 2007.

[36] L.P. Wang, S. Chen, and J.G. Brasseur. Examination of the hypothesis in the Kolmogorov refined turbulence theory through high resolution simulations. Part 2. Passive scalar fields. Journal of Fluid Mechanics, 400:163-197, 1999. 